\title{
Members of Centaurus OB1 and NGC 4755: new spectroscopic and astrometric studies $\star, \star \star$
}

\author{
M. A. Corti ${ }^{1,2}$ and R. B. Orellana ${ }^{2,3}$ \\ ${ }^{1}$ Instituto Argentino de Radioastronomía, CCT-La Plata, CONICET, CC No. 5, 1894 Villa Elisa, Argentina \\ e-mail: mariela@fcaglp.unlp.edu.ar \\ 2 Facultad de Ciencias Astronómicas y Geofísicas, Universidad Nacional de La Plata, Paseo del Bosque s/n, 1900 La Plata, Argentina \\ 3 Instituto de Astrofísica de La Plata, CCT-La Plata, CONICET, Argentina
}

Received 15 November 2012 / Accepted 26 February 2013

\section{ABSTRACT}

\begin{abstract}
Context. An OB association is a gravitationally unbound group of stars. The identification of the members of the association is an important aspect of its study. There are few astrometric membership studies for OB associations, but the combination of these studies with astrophysical techniques becomes a very powerful tool for this type of research.

Aims. We perform new spectroscopic and astrometric studies of the members of the association Centaurus OB1 (Cen OB1) and the open cluster NGC 4755.

Methods. To carry out this study we used new spectroscopic optical observations obtained at CASLEO, and proper motion and photometric data from the Tycho-2 Catalogue and other databases available via the Internet.

Results. With this investigation we found 52 members of Cen OB1 $\left(l=303^{\circ} .7, b=00^{\circ}\right)$ and four members of NGC $4755\left(l=303^{\circ} .2\right.$, $b=2.5$ ), an open cluster integrated into the Cen OB1 stellar association. The distance, heliocentric radial velocity, proper motion, and age of Cen OB1 are $2.6 \pm 0.5 \mathrm{kpc}, \sim-20 \mathrm{~km} \mathrm{~s}^{-1}, \mu_{\alpha} \cos \delta=-4.78 \pm 0.10 \mathrm{mas} \mathrm{yr}^{-1}, \mu_{\delta}=-0.93 \pm 0.10 \mathrm{mas} \mathrm{yr}^{-1}$, and $\sim 20 \times 10^{6}$ years old, respectively. The same parameters found for NGC 4755 are $1.9 \pm 0.5 \mathrm{kpc},-18 \pm 2 \mathrm{~km} \mathrm{~s}^{-1}, \mu_{\alpha} \cos \delta=-4.14 \pm 0.19 \mathrm{mas} \mathrm{yr}^{-1}$, $\mu_{\delta}=-1.09 \pm 0.12$ mas $^{-1} r^{1}$, and $\sim 15 \times 10^{6}$ years old. The pulsars PSR J1253-5820 and PSR J1254-6150 could have been born as the result of supernovae explosions that occurred in the past by massive members of Cen OB1. We have seen Infrared Astronomical Satellite (IRAS) sources near Cen OB1, that show that star formation is still occurring. The age gradient observed between the different members of Cen OB1 makes it likely that the stellar association and IRAS sources represent different stages of a sequential formation process.
\end{abstract}

Key words. stars: early-type - proper motions - open clusters and associations: individual: Centaurus OB1 open clusters and associations: individual: NGC 4755

\section{Introduction}

An OB association is a young star group ( $\leq 50 \mathrm{Myr})$ of low density which has a significant population of $\mathrm{O}$ and $\mathrm{B}$ stars. It is believed that they originated as a group which was born in a relatively small region of space within a giant molecular cloud. After the removal of the gas and the dust, the stellar association is transformed into a gravitationally unbound association and it expands. Its projected dimensions cover a range of $\sim 10$ a $\sim 100$ pc (Brown et al. 1999).

Among all the reasons that make the study of $\mathrm{OB}$ associations important, it is the capacity of its large-mass members of causing a big impact in the evolution of the interstellar medium and the creation of neutral hydrogen (HI) shell that are the most significant.

As part of a project in which the investigation of the possible link between star sets and HI shells is proposed, the HI GS305+04-26, centred in $(l, b)=\left(305^{\circ},+4^{\circ}\right)$ (Corti et al. 2012) was investigated. In a study of this kind it was determined that the HI GS305+04-26, could have been generated by the open cluster NGC 4755 and the stellar association Cen OB1. To study the possible generator of the HI GS305+04-26, it was necessary to identify the members of NGC 4755 and Cen OB1.

\footnotetext{
* Based on data results from observations made with CASLEO, Argentina.

$\star \star$ Table 5 is available in electronic form at http://www. aanda.org
}

Studies made so far to NGC 4755 and Cen OB1 were:

- Humphreys (1978) was the first to publish a list of members of Cen OB1 as a result of a spectral-photometric study and obtained 29 possible members, from which 7 stars would belong to the open cluster NGC 4755.

- Humphreys \& McElroy (1984), based on photometric work, published a catalogue of Cen OB1 with 106 members.

- Baumgardt et al. (2000) discussed the membership of NGC 4755 using parallaxes, proper motion data and photometry data from the HIPPARCos Catalogue and found 7 members.

- McClure-Griffiths et al. (2001) incorporated the star Wolf-

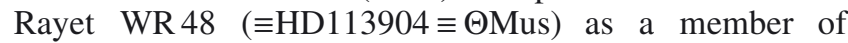
Cen OB1.

- More recently, Kharchenko et al. (2005) estimated the mean absolute proper motion of NGC 4755 and found 15 members using the ASCC-2.5 Catalogue data. The distance estimated was $1976 \mathrm{pc}$.

- Corti et al. (2012) incorporated the PSRJ 1253-5820 and PSRJ 1254-6150 pulsars as members of Cen OB1. Both could have arisen as a result of the explosion as supernovae $(\mathrm{SNe})$ of two stars that are possible members of Cen OB1.

Upon consulting these works, it is evident that there are no astrometric studies on the Cen OB1 association, and there are a few 
Table 1. NGC 4755 data available in the literature.

\begin{tabular}{|c|c|c|c|c|c|c|c|c|c|}
\hline Author & $\begin{array}{l}\alpha_{J 2000.0} \\
\text { (h:m:s) }\end{array}$ & $\begin{array}{c}\delta_{J 2000.0} \\
\left({ }^{\circ}:^{\prime}:^{\prime \prime}\right)\end{array}$ & $\begin{array}{l}R_{\mathrm{c}} \\
\left({ }^{\prime}\right)\end{array}$ & $\begin{array}{c}\mu_{\alpha} \cos \delta \\
\left(\mathrm{mas} \mathrm{yr}^{-1}\right)\end{array}$ & $\begin{array}{c}\mu_{\delta} \\
\left(\operatorname{mas~yr}^{-1}\right)\end{array}$ & $\begin{array}{c}\mathrm{RV} \\
\left(\mathrm{km} \mathrm{s}^{-1}\right)\end{array}$ & $N_{1}$ & $\begin{array}{c}D \\
(\mathrm{pc})\end{array}$ & $\log (t)$ \\
\hline Baumgardt et al. (2000) & $12: 53: 39$ & $-60: 21: 42$ & 5 & $-4.42 \pm 0.33$ & $-1.53 \pm 0.30$ & $+20.0 \pm 3.0$ & 5 & 1976 & 7.22 \\
\hline Dias et al. (2002) & 12:53:39 & $-60: 21: 42$ & 6 & $-5.35 \pm 0.25$ & $-0.90 \pm 0.25$ & $(\ldots)$ & 192 & $(\ldots)$ & $(\ldots)$ \\
\hline Kharchenko et al. (2005) & $12: 53: 36$ & $-60: 22: 00$ & 15 & $-4.30 \pm 0.26$ & $-1.17 \pm 0.31$ & $-12.5 \pm 3.2$ & 15 & 1976 & 6.98 \\
\hline This paper & $12: 53: 30$ & $-60: 22: 00$ & 11 & $-4.14 \pm 0.19$ & $-1.09 \pm 0.12$ & $-18 \pm 2$ & 4 & 1888 & 7.18 \\
\hline
\end{tabular}

works for the open cluster NGC 4755 (Baumgardt et al. 2000; Kharchenko et al. 2005) which do not agree regarding the membership assigned to it. In this paper, we propose to revisit the region of Cen OB1 and NGC 4755, using the astrometric and photometric data of the Tycho-2 Catalogue and spectro-photometric data obtained from Simbad and our own data.

\section{Observational data}

This research was carried out using new observational data and databases publicly available, namely spectroscopic observations and astrometric and photometric data.

\subsection{Spectroscopic observations}

Spectroscopic observations of 31 stars that are possible members of Cen OB1 were obtained at Complejo Astronómico El Leoncito (CASLEO) ${ }^{1}$ during April 2009. The spectra were obtained using the REOSC Cassegrain echelle spectrograph attached to the 2.15-m Jorge Sahade telescope. The detector was a TEK CCD $(1024 \times 1024$ pixels $)$ which has a pixel size of $24 \mu \mathrm{m}$. A grating of $400 \mathrm{l} / \mathrm{mm}$ was used as a cross disperser, and the slit width was set either to $250 \mu \mathrm{m}$ or $300 \mu \mathrm{m}$. The reciprocal dispersion was $0.2 \AA \mathrm{px}^{-1}$. These spectra covered the wavelength range from $3800 \AA$ to $6500 \AA$, and the signal-to-noise ratio was $20<S / N<50$.

Thorium-argon (Th-Ar) comparison arc images used for the wavelength calibration in stellar spectra were observed at the same sky position as that of the stellar images, immediately after or before the stellar exposures. Bias frames were also obtained every night, as well as spectra of the stars HR 2806 and HR 7773 as radial velocity standards. The spectra of several standard spectral types (SpTs) were also obtained during the observing run. All spectra were processed and analysed using the IRAF $^{2}$ package. SpTs and radial velocities from the stars that could not be observed at the CASLEO were obtained from the SIMBAD Astronomical Database ${ }^{3}$ (for references see Table 5).

\subsection{Astrometric and photometric data}

The astrometric and photometric data used in this paper come from the Tycho-2 Catalogue with position, proper motions, and two-colour photometry for 2.5 million stars covering the whole sky (Høg et al. 2000b). The Tycho-2 Catalogue is the first densification of the HIPPARCos frame. The proper motions result from the combination with the re-analysis of 144 additional astrometric catalogues. A detailed description of the construction of the Tycho-2 Catalogue can be found in Høg et al. (2000a).

\footnotetext{
1 Operated under agreement among CONICET, SeCyT, and the Universities of La Plata, Córdoba and San Juan, Argentina.

2 IRAF is distributed by NOAO, operated by AURA, Inc. under an agreement with NSF.

3 http://cdsportal.u-strasbg.fr/
}

For the faintest stars in common with Tycho-1, Tycho-2 has better positional and photometric accuracy. This is because the new method of reduction applied to the HIPPARCOS data uses photon superposition for each star in the ensemble of observations made during the entire mission instead of treating each observation separately.

The Tycho-2 Catalogue presents very precise proper motions. The internal or random errors are generally between 1

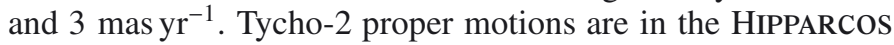
system; there are no significant systematic differences between the proper motions of the two catalogues, as shown by Urban et al. (2000). The systematic errors under $0.5 \mathrm{mas} \mathrm{yr}^{-1}$ are typically $10 \%$ of the random errors, and are almost equal to the formal errors for the rotation of the HIPPARCos proper motion with respect to the International Celestial Reference System (ICRS),

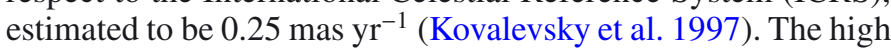
quality of the Tycho- 2 proper motions and their agreement with the HIPPARCos set allow the determination of the mean proper motions and the membership of open clusters and associations.

\section{Results}

\subsection{Astrometric analysis}

\subsubsection{NGC 4755}

As a first step, we identified the cluster members. Several studies have shown different results for the open cluster NGC 4755 (Table 1) (Baumgardt et al. 2000; Dias et al. 2002; Kharchenko et al. 2005), where $\alpha_{J 2000.0}$ (in hours) and $\delta_{J 2000.0}$ (in degrees) are the coordinates of the cluster's centre, $R_{\mathrm{c}}$ is the radius in arcmin, $\mu_{\alpha} \cos \delta$ and $\mu_{\delta}$ are the components of its mean absolute proper motion in mas $\mathrm{yr}^{-1}$, RV is the radial velocity in $\mathrm{km} \mathrm{s}^{-1}, N_{1}$ is the number of the members, $D$ is the distance in parsec, and $t$ in years represents the age.

We selected the stars from the Tycho-2 Catalogue centred at $\alpha=12: 53.5, \delta=-60: 22$ in a circular region of radius $20^{\prime}$ containing 55 stars. An over-density is expected to be found in the sky as well as in the vector point diagram (VPD) for cluster stars. It is possible to analyse these over-densities adopting the mathematical model suggested by Vasilevskis et al. (1958) and the technique based upon the maximum likelihood principle developed by Sanders (1971). The number of stars of this circular region $N=55$ had been expressed as

$N=N_{1}+N_{2}$

where $N_{1}$ is the number of the cluster members and $N_{2}$ is the number of the field stars. Jones \& Walker (1988) improved the method using an exponential function $\rho_{1}(\mathrm{r})$ to describe the areal stellar density for the cluster stars according to van den Bergh \& Sher (1960). The stellar radial density profile was obtained by counting the number of stars within concentric annuli around the cluster centre. This profile was modeled by the overlapping of two functions

$\rho(r)=\rho_{1}(r)+\rho_{2}$ 
where $\rho_{1}(r)$ is the cluster stellar density, $\rho_{2}$ is the field stellar density and $\rho_{2}$ is a constant. The cluster stellar density is represented by an exponential function

$\rho_{1}(r)=\rho_{0} \exp \left(-r / r_{0}\right)$

where $\rho_{0}$ is the central cluster stellar density, $r_{0}$ is the characteristic radius, and $r$ the distance from the cluster's centre.

The proper motion distribution of the region selected consisted in the overlapping of two bivariate normal frequency functions

$\Phi_{i}\left(\mu_{x i}^{\mathrm{c}}, \mu_{y i}^{\mathrm{c}}, r\right)=\phi_{1 i}\left(\mu_{x i}^{\mathrm{c}}, \mu_{y i}^{\mathrm{c}}, r\right)+\phi_{2 i}\left(\mu_{x i}^{\mathrm{c}}, \mu_{y i}^{\mathrm{c}}\right)$

where $\phi_{1 i}$ is a circular distribution for cluster stars and $\phi_{2 i}$ is an elliptical distribution for field stars; $\mu_{x i}^{\mathrm{c}}, \mu_{y i}^{\mathrm{c}}$ are the ith star proper motion in $\alpha \cos \delta$ and $\delta$, respectively. These coordinate axes are coincident with the field distribution axes after rotating the VPD by an angle $\theta$.

The circular and elliptical distributions have taken the form

$$
\begin{aligned}
\phi_{1 i}\left(\mu_{x i}^{\mathrm{c}}, \mu_{y i}^{\mathrm{c}}, r\right)= & \frac{\rho_{1}(r)}{2 \pi \sigma_{\mathrm{c}}^{2}} \\
& \times \exp \left[-\frac{\left(\mu_{x i}^{\mathrm{c}}-\mu_{x \mathrm{c}}\right)^{2}+\left(\mu_{y i}^{\mathrm{c}}-\mu_{y \mathrm{c}}\right)^{2}}{2 \sigma_{\mathrm{c}}^{2}}\right]
\end{aligned}
$$

and

$$
\begin{aligned}
\phi_{2 i}\left(\mu_{x i}^{\mathrm{c}}, \mu_{y i}^{\mathrm{c}}\right)= & \frac{\rho_{2}}{2 \pi \sigma_{x \mathrm{f}}^{\mathrm{c}} \sigma_{y \mathrm{f}}^{\mathrm{c}}} \\
& \times \exp \left[-\frac{\left(\mu_{x i}^{\mathrm{c}}-\mu_{x \mathrm{f}}^{\mathrm{c}}\right)^{2}}{2\left(\sigma_{x \mathrm{f}}^{\mathrm{c}}\right)^{2}}-\frac{\left(\mu_{y i}^{\mathrm{c}}-\mu_{y \mathrm{f}}^{\mathrm{c}}\right)^{2}}{2\left(\sigma_{y \mathrm{f}}^{\mathrm{c}}\right)^{2}}\right]
\end{aligned}
$$

$\sigma_{x \mathrm{f}}^{\mathrm{c}}, \sigma_{y \mathrm{f}}^{\mathrm{c}}$ being the field elliptical dispersions, $\mu_{x \mathrm{f}}^{\mathrm{c}}, \mu_{y \mathrm{f}}^{\mathrm{c}}$ the field mean proper motion, $\sigma_{\mathrm{c}}$ the cluster circular dispersion and $\mu_{x \mathrm{c}}$, $\mu_{y \mathrm{c}}$ the cluster mean proper motion.

The parameters $\rho_{0}, r_{0}$, and $\rho_{2}$ were obtained by fitting Eq. (2) to the radial stellar density profile. The other seven parameters present in Eqs. (5) and (6) can be found by applying the maximum likelihood principle. The unknown cluster parameters, therefore, can be entirely determined.

The probability for the ith star has been calculated as

$P_{\mathrm{ci}}\left(\mu_{x i}^{\mathrm{c}}, \mu_{y i}^{\mathrm{c}}, r\right)=\frac{\phi_{1 i}\left(\mu_{x i}^{\mathrm{c}}, \mu_{y i}^{\mathrm{c}}, r\right)}{\Phi_{i}\left(\mu_{x i}^{\mathrm{c}}, \mu_{y i}^{\mathrm{c}}, r\right)}$.

A cluster member is found when $P_{\mathrm{ci}}>0.5$.

To apply the model described, we analysed the proper motion data in an elliptical subregion centred in $\mu_{\alpha} \cos \delta=$ -6.5 mas yr$^{-1}, \mu_{\delta}=-2.5$ mas yr $^{-1}$, with semi-major and semiminor axes 8 and 5 mas $\mathrm{yr}^{-1}$, respectively, of the VPD containing 42 stars, where $\theta=75.33$ (see Fig. 1). The function $\rho(r)$ given in Eq. (2) was adjusted to the radial stellar density profile, as can be seen in Fig. 2, with the following parameters: $\rho_{0}=1.558 \pm 0.437 \mathrm{stars} /\left(^{\prime}\right)^{2}, r_{0}=1.212 \pm 0.186$, and $\rho_{2}=$ $0.027 \pm 0.006$ stars $/\left({ }^{\prime}\right)^{2}$

After applying the method of maximum likelihood to Eqs. (5) and (6), the cluster parameters were $\mu_{x \mathrm{c}}=$ $-4.14 \pm 0.19$ mas yr$^{-1}, \mu_{y c}=-1.09 \pm 0.12 \operatorname{mas~yr}^{-1}, \sigma=$ $0.63 \pm 0.28 \mathrm{mas} \mathrm{yr}^{-1}$, and the field parameters $\mu_{x \mathrm{f}}^{\mathrm{c}}=$ $-4.69 \pm 0.57$ mas yr $^{-1}, \mu_{y \mathrm{f}}^{\mathrm{c}}=-1.32 \pm 0.53 \mathrm{mas} \mathrm{yr}^{-1}, \sigma_{x \mathrm{f}}^{\mathrm{c}}=1.60 \pm$ $0.45 \mathrm{mas} \mathrm{yr}^{-1}$, and $\sigma_{y \mathrm{f}}^{\mathrm{c}}=2.05 \pm 0.51 \mathrm{mas} \mathrm{yr}^{-1}$. Once the cluster and field parameters have been determined, the membership probability of the ith star was given by Eq. (7).

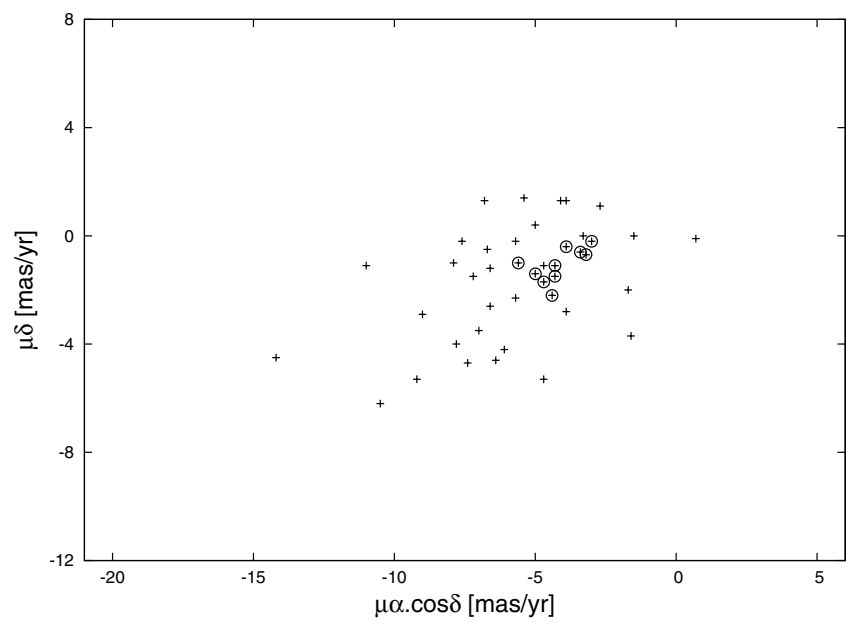

Fig. 1. Vector point diagram (VPD) of NGC 4755. Open circles around the crosses represent the astrometric cluster members, and crosses the rest of the stars of the region.

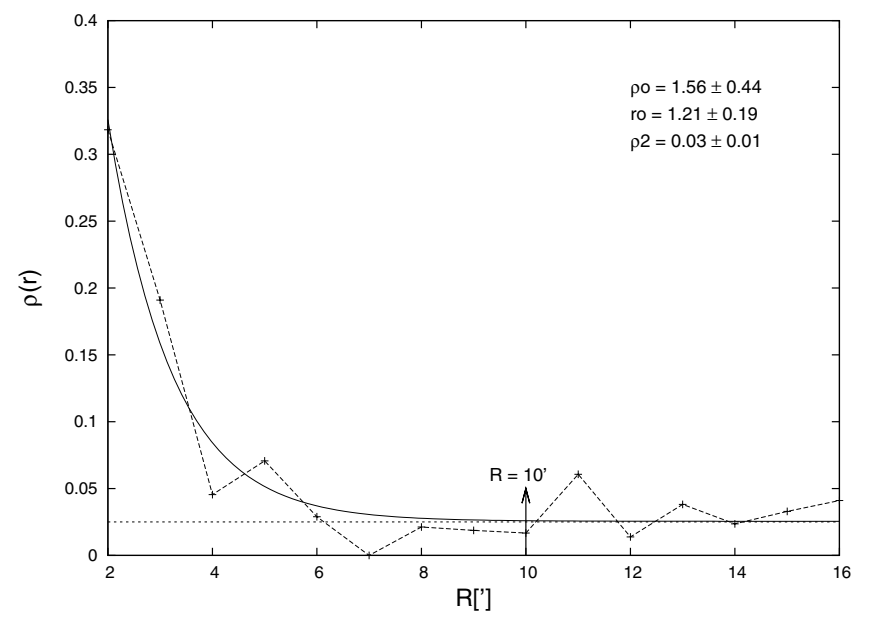

Fig. 2. Stellar density profiles in the region of NGC 4755 as a function of the distance from the cluster centre.

Among the 55 stars in the region of NGC 4755, 10 stars with higher than 0.5 probabilities have been found and should be considered as the astrometric members of the cluster. They are located in a circular region 10'8 from the cluster centre. Figure 1 shows the location of the 10 cluster members in the VPD.

The following information on the astrometric cluster members is shown in Table 2: ID is the identification of the star in some catalogues; $r$ is the distance from the cluster centre in arcmin; $(\alpha, \delta)$ are the coordinates in hours and degrees, respectively; $\mu_{\alpha} \cos \delta$ and $\mu_{\delta}$ are the components of the proper motion in mas $\mathrm{yr}^{-1} ; P_{\mathrm{c}}$ is the probability obtained; TYC is Tycho's number; $B_{\mathrm{T}}$ and $V_{\mathrm{T}}$ are Tycho- 2 magnitudes.

Finally, based on the calculations of distance obtained as described in Sect. 3.2.1, it can be seen that there are four possible members of NGC 4755. The information is given in Table 3, where the $V$ and $(B-V)$ are the magnitude and colour, respectively, corrected from the Tycho-2 system to the Johnson system (Bessell \& Brett 1988); RV is the heliocentric radial velocity; and DM the distance modulus of the Sun.

\subsubsection{Cen $\mathrm{OB} 1$}

Previous spectrophotometric studies suggested the existence of a stellar association in addition to an open cluster in the region 
Table 2. Astrometric cluster members of NGC 4755.

\begin{tabular}{lccccccccc}
\hline \hline ID & $\begin{array}{c}r \\
\left({ }^{\prime}\right)\end{array}$ & $\begin{array}{c}\alpha \\
(\mathrm{h}: \mathrm{m}: \mathrm{s})\end{array}$ & $\begin{array}{c}\delta \\
\left({ }^{\circ}:^{\prime \prime}\right)\end{array}$ & $\begin{array}{c}\mu_{\alpha} \cos \delta \\
\left(\mathrm{mas} \mathrm{yr}^{-1}\right)\end{array}$ & $\begin{array}{c}\mu_{\delta} \\
\left(\mathrm{mas} \mathrm{yr}^{-1}\right)\end{array}$ & $P_{\mathrm{c}}$ & TYC & $\begin{array}{c}B_{\mathrm{T}} \\
(\mathrm{mag})\end{array}$ & $\begin{array}{c}V_{\mathrm{T}} \\
(\mathrm{mag})\end{array}$ \\
\hline HD 111934 & 1.1048 & $12: 50: 37.5$ & $-60: 05: 08$ & -5.6 & -1.0 & 0.62 & $8989-3109-1$ & 7.07 & 6.89 \\
$(\ldots)$ & 1.3809 & $12: 50: 39.1$ & $-60: 04: 56$ & -3.0 & -0.2 & 0.69 & $8989-3109-2$ & 8.35 & 8.22 \\
CD-59 4459 & 1.7421 & $12: 50: 41.2$ & $-60: 04: 41$ & -3.9 & -0.4 & 0.75 & $8989-3108-1$ & 9.61 & 7.59 \\
HD 111904 & 2.5004 & $12: 50: 21.9$ & $-60: 03: 26$ & -3.2 & -0.7 & 0.63 & $8989-2110-1$ & 6.11 & 5.80 \\
CD-59 4552 & 3.0040 & $12.50: 46.3$ & $-60: 07: 56$ & -5.0 & -1.4 & 0.62 & $8989-2130-1$ & 8.37 & 8.25 \\
HD 312081 & 5.6883 & $12: 50: 59.4$ & $-60: 10: 04$ & -4.3 & -1.1 & 0.82 & $8989-1657-1$ & 11.62 & 10.12 \\
HD 312075 & 7.5521 & $12: 49: 36.7$ & $-60: 02: 08$ & -4.3 & -1.5 & 0.79 & $8989-2518-1$ & 10.84 & 10.62 \\
HD 312082 & 8.4266 & $12: 51: 32.4$ & $-60: 08: 57$ & -4.4 & -2.2 & 0.52 & $8989-1968-1$ & 12.49 & 10.58 \\
$(\ldots)$ & 10.6372 & $12: 51: 27.7$ & $-60: 13: 34$ & -3.4 & -0.6 & 0.69 & $8989-2309-1$ & 12.42 & 12.36 \\
$(\ldots)$ & 10.7645 & $12: 51: 54.9$ & $-60: 03: 49$ & -4.7 & -1.7 & 0.67 & $8989-2270-1$ & 12.46 & 12.14 \\
\hline
\end{tabular}

Table 3. Possible members of NGC 4755.

\begin{tabular}{ccccccccc}
\hline \hline ID & $\begin{array}{c}\alpha_{J 2000.0} \\
(\mathrm{~h}: \mathrm{m}: \mathrm{s})\end{array}$ & $\begin{array}{c}\delta_{J 2000.0} \\
\left(\circ:^{\prime}:^{\prime \prime}\right.\end{array}$ & $\begin{array}{c}V \\
(\mathrm{mag})\end{array}$ & $\begin{array}{c}(B-V) \\
(\mathrm{mag})\end{array}$ & SpT & TYC & $\begin{array}{c}\text { RV } \\
\left(\mathrm{km} \mathrm{s}^{-1}\right)\end{array}$ & DM \\
\hline HD 111904 & $12: 53: 21.9$ & $-60: 19: 43$ & 5.8 & 0.3 & $\mathrm{~B}^{2} \mathrm{Ia}^{1}$ & $8989-2110-1$ & $-19^{1}$ & 12.0 \\
HD 111934 & $12: 53: 37.6$ & $-60: 21: 25$ & 6.9 & 0.1 & $\mathrm{~B}^{1} \mathrm{Ib}^{1}$ & $8989-3109-1$ & $-20(2)^{1}$ & 11.0 \\
$(\ldots)$ & $12: 53: 39.2$ & $-60: 21: 13$ & 8.2 & 0.1 & $(\ldots)$ & $8989-3109-2$ & $(\ldots)$ & 11.0 \\
$\mathrm{CP}-594552$ & $12: 53: 46.5$ & $-60: 24: 12$ & 8.3 & 0.1 & $\mathrm{~B} 1 \mathrm{III}^{3}$ & $8989-2130-1$ & $-15^{2}$ & 11.5 \\
\hline
\end{tabular}

References. ${ }^{(1)}$ This paper. ${ }^{(2)}$ Kharchenko et al. (2007). ${ }^{(3)}$ Kharchenko \& Roeser (2009).

of Centaurus (Humphreys 1978; Humphreys \& McElroy 1984; McClure-Griffiths et al. 2001). To perform an identification of astrometric members, we selected the stars from the Tycho-2 Catalogue centred at $\alpha=12: 58: 30, \delta=-62: 30: 00$ in a square area of $7^{\circ}$ containing 898 stars. Then, an over-density in the VPD caused by the low values of the velocity dispersions of the members is expected to be found if there is an association. Therefore, a selection based on proper motion is the most reliable method for the separation of the association from field stars. We analysed this over-density using the model proposed by Orellana et al. (2010). In their paper they proposed for the first time, a standardised way to identify the members of an association. To do this, the authors applied the same technique used for open clusters considering only the proper motions based upon the maximum likelihood principle (Sanders 1971).

To find the members of the association, cluster members were taken out. The new subregion has a total population of 884 stars. So, the proper motion distribution in the new subregion took the form

$\Psi_{i}\left(\mu_{x i}^{\mathrm{a}}, \mu_{y i}^{\mathrm{a}}\right)=\psi_{1 i}\left(\mu_{x i}^{\mathrm{a}}, \mu_{y i}^{\mathrm{a}}\right)+\psi_{2 i}\left(\mu_{x i}^{\mathrm{a}}, \mu_{y i}^{\mathrm{a}}\right)$

where

$$
\begin{aligned}
\psi_{1 i}\left(\mu_{x i}^{\mathrm{a}}, \mu_{y i}^{\mathrm{a}}\right)= & \frac{N_{3}}{2 \pi \sigma_{\mathrm{a}}^{2}} \\
& \times \exp \left[-\frac{\left(\mu_{x i}^{\mathrm{a}}-\mu_{x \mathrm{a}}\right)^{2}+\left(\mu_{y i}^{\mathrm{a}}-\mu_{y \mathrm{a}}\right)^{2}}{2 \sigma_{\mathrm{a}}^{2}}\right]
\end{aligned}
$$

and

$$
\begin{aligned}
\psi_{2 i}\left(\mu_{x i}^{\mathrm{a}}, \mu_{y i}^{\mathrm{a}}\right)= & \frac{N_{4}}{2 \pi \sigma_{x \mathrm{f}}^{\mathrm{a}} \sigma_{y \mathrm{f}}^{\mathrm{a}}} \\
& \times \exp \left[-\frac{\left(\mu_{x i}^{\mathrm{a}}-\mu_{x \mathrm{f}}^{\mathrm{a}}\right)^{2}}{2\left(\sigma_{x \mathrm{f}}^{\mathrm{a}}\right)^{2}}-\frac{\left(\mu_{y i}^{\mathrm{a}}-\mu_{y \mathrm{f}}^{\mathrm{a}}\right)^{2}}{2\left(\sigma_{y \mathrm{f}}^{\mathrm{a}}\right)^{2}}\right] .
\end{aligned}
$$

The symbols $\sigma_{x \mathrm{f}}^{\mathrm{a}}, \sigma_{y \mathrm{f}}^{\mathrm{a}}$ are the elliptical dispersions for the field stars, $\sigma_{\mathrm{a}}$ the circular dispersion for the association stars, $\mu_{x \mathrm{f}}^{\mathrm{a}}, \mu_{y \mathrm{f}}^{\mathrm{a}}$ the field star mean proper motion, and $\mu_{x \mathrm{a}}, \mu_{y \mathrm{a}}$ the association mean proper motion. $N_{3}$ is the number of the association members, and $N_{4}$ the number of the field stars. The parameters present in Eqs. (9) and (10) would be found by applying the maximum likelihood principle. It determines the association and field unknowns. The probability for the ith star has been calculated as

$P_{\mathrm{a} i}\left(\mu_{x i}^{\mathrm{a}}, \mu_{y i}^{\mathrm{a}}\right)=\frac{\psi_{1 i}\left(\mu_{x i}^{\mathrm{a}}, \mu_{y i}^{\mathrm{a}}\right)}{\Psi_{i}\left(\mu_{x i}^{\mathrm{a}}, \mu_{y i}^{\mathrm{a}}\right)}$.

Then, we analysed the proper motion data of an elliptical subregion centred in $\mu_{\alpha} \cos \delta=0.0 \mathrm{mas} \mathrm{yr}^{-1}, \mu_{\delta}=0.0 \mathrm{mas} \mathrm{yr}^{-1}$,

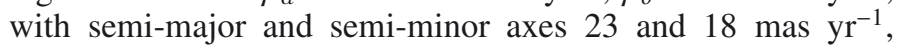
respectively, of VPD containing 661 stars, where $\theta=45^{\circ} .75$. We applied the method of the maximum likelihood to Eqs. (9) and (10). The association parameters were found to be $N_{3}=299$, $\mu_{x \mathrm{a}}=-5.40 \pm 0.18 \operatorname{mas~yr}^{-1}, \mu_{y \mathrm{a}}=-1.04 \pm 0.16 \mathrm{mas} \mathrm{yr}^{-1}$, $\sigma_{\mathrm{a}}=1.89 \pm 0.11 \mathrm{mas} \mathrm{yr}^{-1}$, and for the field stars $N_{4}=362$, $\mu_{x \mathrm{f}}^{\mathrm{a}}=-6.82 \pm 0.84 \mathrm{mas} \mathrm{yr}^{-1}, \mu_{y \mathrm{f}}^{\mathrm{a}}=3.41 \pm 0.89 \mathrm{mas} \mathrm{yr}^{-1}$, $\sigma_{x \mathrm{f}}^{\mathrm{a}}=5.99 \pm 0.35 \mathrm{mas} \mathrm{yr}^{-1}$, and $\sigma_{y \mathrm{f}}^{\mathrm{a}}=6.40 \pm 0.37 \mathrm{mas} \mathrm{yr}^{-1}$. Figures $3 \mathrm{a}$ and $4 \mathrm{a}$ show the location of the 299 association members in the spatial distribution and in the VPD, respectively, with open circles around the dots.

Next, we removed the contamination produced by the field stars located at different distances. For this we inspected the $B_{\mathrm{T}}$ and $V_{\mathrm{T}}$ of the 299 astrometric member stars whose DM were calculated following the procedure in Sect. 3.2.1. and are shown in Fig. 5. We can see a concentration of 86 stars for DM = $12 \pm 0.6$ which corresponds to a distance $\mathrm{D}=2588 \pm 534 \mathrm{pc}$. Figure $4 \mathrm{~b}$ shows the 86 association members. Further adjustment was made by applying the Orellana et al. (2010) model by eliminating from the initial group of 299 stars the 213 that are not distance members. The new parameters are $N_{3}=52$, $\mu_{x \mathrm{a}}=-4.78 \pm 0.10 \mathrm{mas} \mathrm{yr}^{-1}, \mu_{y \mathrm{a}}=-0.93 \pm 0.10 \mathrm{mas} \mathrm{yr}^{-1}$, $\sigma_{\mathrm{a}}=1.05 \pm 0.14 \mathrm{mas} \mathrm{yr}^{-1}$, and for the field stars $N_{4}=609$, $\mu_{x \mathrm{f}}^{\mathrm{a}}=-5.58 \pm 0.62 \operatorname{mas~yr}^{-1}, \mu_{y \mathrm{f}}^{\mathrm{a}}=-2.02 \pm 0.43 \mathrm{mas} \mathrm{yr}^{-1}$, $\sigma_{x \mathrm{f}}^{\mathrm{a}}=6.59 \pm 0.27 \mathrm{mas} \mathrm{yr}^{-1}$ and $\sigma_{y \mathrm{f}}^{\mathrm{a}}=7.57 \pm 0.31 \mathrm{mas} \mathrm{yr}^{-1}$. 
M. A. Corti and R. B. Orellana: Members of Centaurus OB1 and NGC 4755: new spectroscopic and astrometric studies
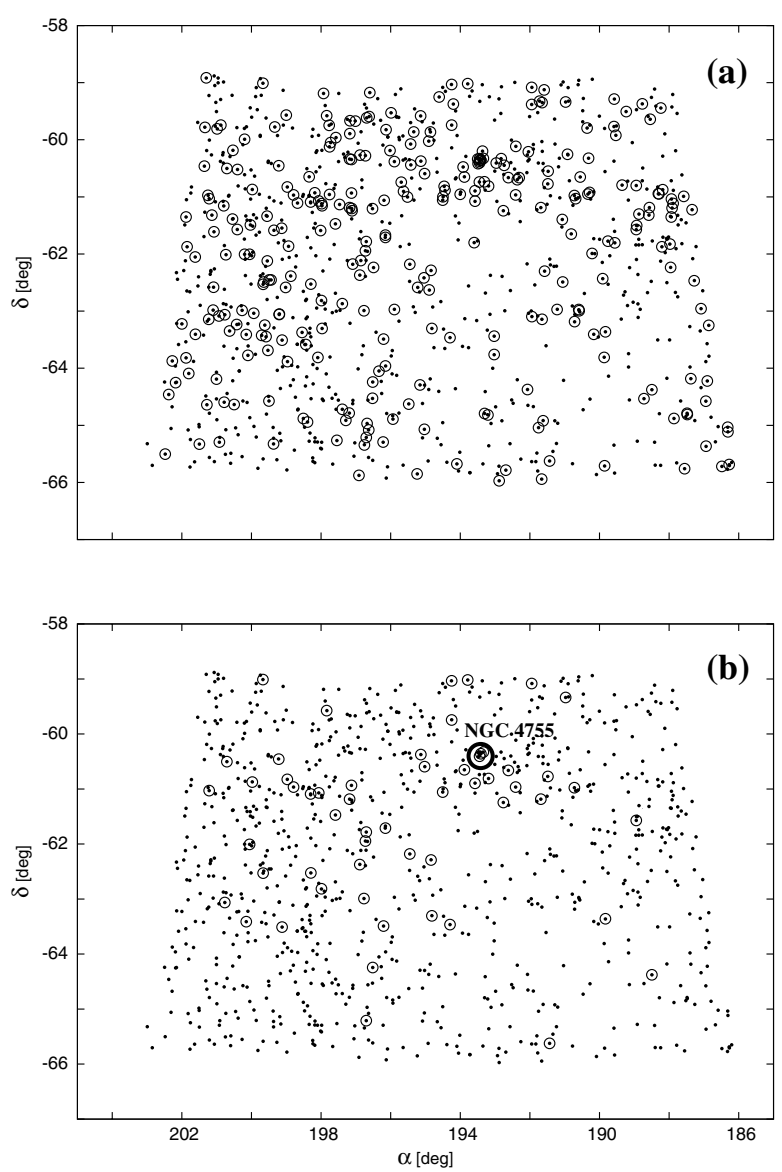

Fig. 3. Spatial distribution of the stellar association Cen OB1. a) Open circles around the dots represent the 299 astrometric association members; the points represent the rest of the stars of the region in the first analysis. b) Open circles represent the 52 astrometric and spectrophotometric association members obtained using the model proposed by Orellana et al. (2010). The NGC 4755 cluster is indicated by the large circle.

A total of 52 stars were identified as likely candidates to be members of Cen OB1. These stars are listed in Table 5 and the spatial distribution and VPD are shown in Figs. $3 b$ and $4 c$, respectively, with open circles around the dots.

\subsection{Spectrophotometric analysis}

\subsubsection{Distance}

The combination of photometric and spectroscopic information provides the possibility of determining individual colour excesses $E(B-V)$ of the program stars. The photometric magnitudes $V_{\mathrm{T}}$ and $B_{\mathrm{T}}$ were corrected from the Tycho- 2 system to the Johnson system (Bessell \& Brett 1988). The intrinsic $(B-V)_{\mathrm{o}}$ colour index and the absolute visual magnitude $M_{\mathrm{v}}$, were obtained from Schmidt-Kaler (1982). The quoted error stems from an assumed $M_{\mathrm{v}}$ uncertainty of $0.5 \mathrm{mag}$ (Walborn 1972). In the $\mathrm{SpT}$ for which $M_{\mathrm{v}}$ was not found, linear interpolation was performed. With these data the visual extinction of each star could be obtained

$A_{\mathrm{v}}=R_{\mathrm{v}} \times E(B-V)$

where $R_{\mathrm{v}}$ is the standard selective absorption coefficient, calculated as

$R_{\mathrm{v}}=3.3+0.2 \times(B-V)_{\circ}+0.04 \times\left((B-V)-(B-V)_{\circ}\right)$.
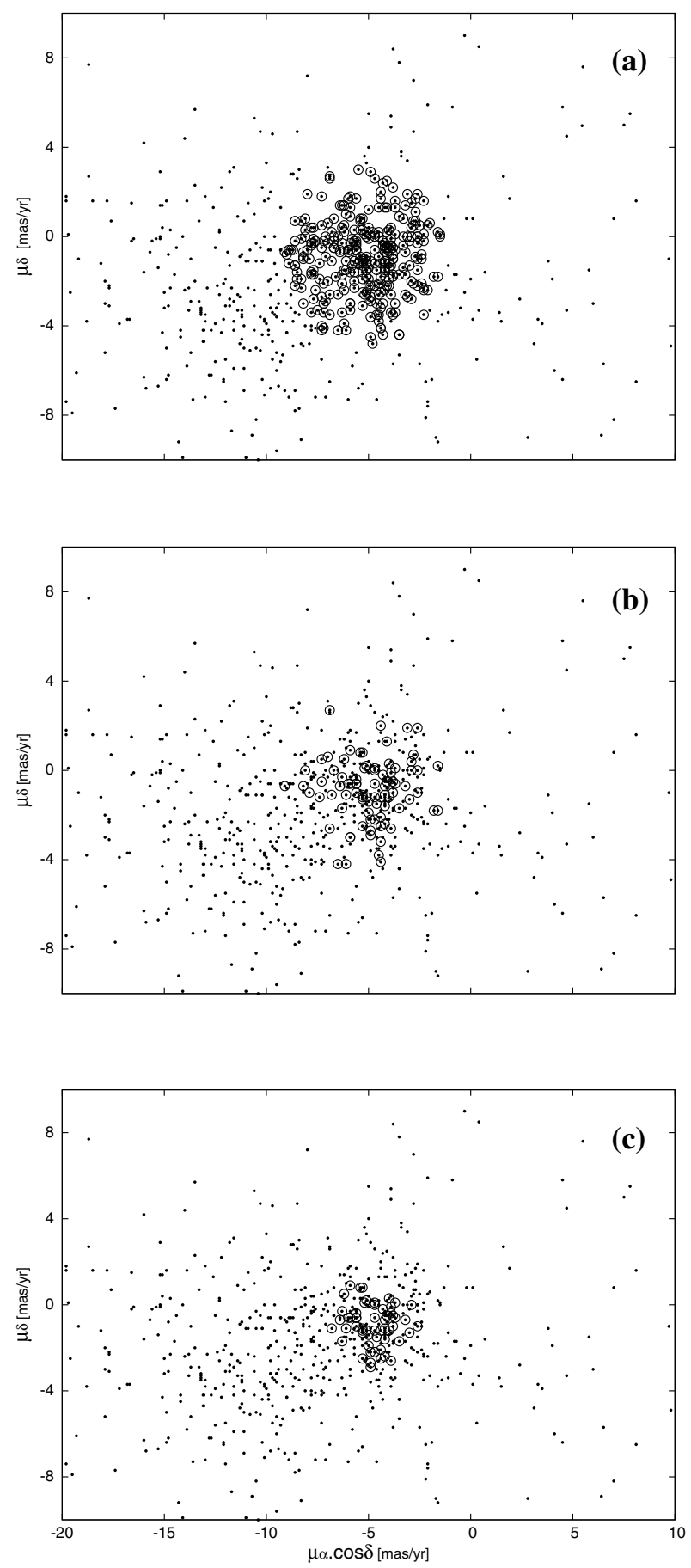

Fig. 4. VPD of the stellar association Cen OB1. a) Open circles around the dots represent the 299 astrometric association members; points represent the rest of the stars of the region in the first analysis. b) Open circles represent the 86 astrometric and spectrophotometric members. c) Open circles represent the 52 astrometric and spectrophotometric association members obtained using the model proposed by Orellana et al. (2010).

Equation (13) was obtained from Schmidt-Kaler (1982) and the value of $R_{\mathrm{v}}$ calculated using Eq. (13) for each star is in the range of $3.25 \leq R_{\mathrm{v}} \leq 3.32$. Then, it was possible to estimate distance moduli

$\mathrm{DM}=V-A_{\mathrm{v}}-M_{\mathrm{v}}=V_{\mathrm{o}}-M_{\mathrm{v}}$.

Figure 5 is the histogram that represents the 299 astrometric member stars of Cen OB1 (Fig. 4a) according to the DM. 


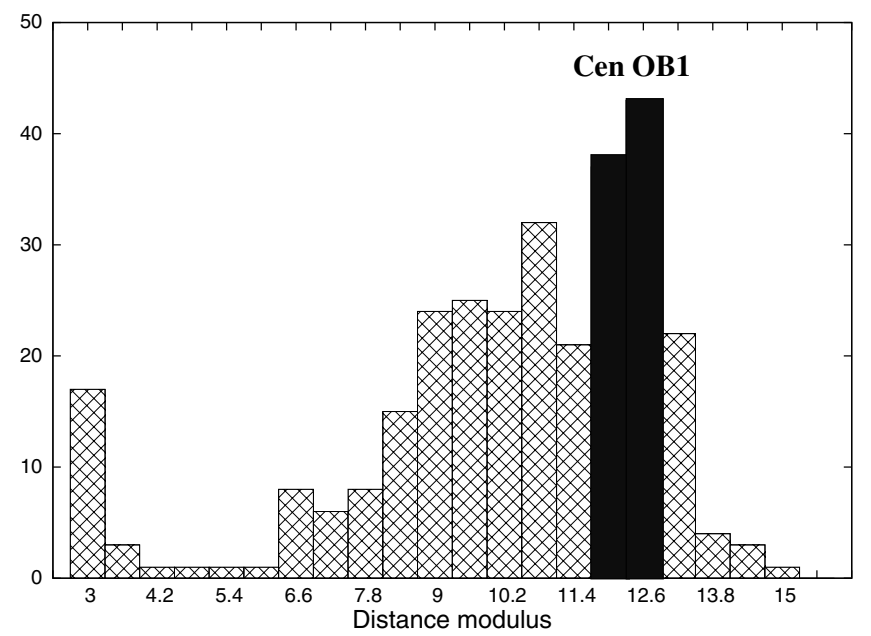

Fig. 5. Distribution of the DM of the 299 astrometric member stars of Cen OB1 (Fig. 4a). The dark colour bins are the 86 stars with $11.4 \leq$ $\mathrm{DM} \leq 12.6$ (Fig. 4b).

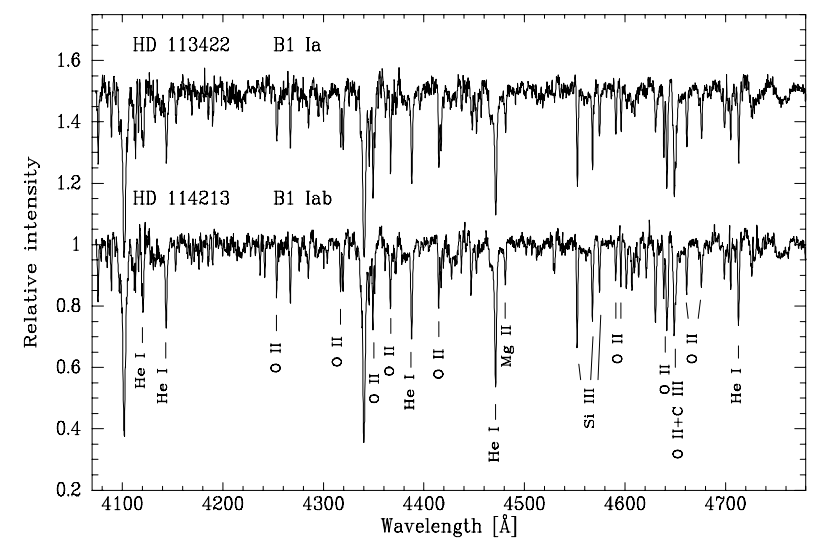

Fig. 6. Blue optical spectra of two B1 supergiant stars, members of Cen OB1. The features indicated are the classification criteria used in the SpTs by the WF90 Catalogue.

This Fig. 5 shows the group of 86 stars with dark colour bins with $11.4 \leq \mathrm{DM} \leq 12.6$ (Fig. 4b). Within both bins there are the 52 stars members of Cen OB1, according to Sect. 3.1.2, with a DM $=12.0 \pm 0.4(2591 \pm 458 \mathrm{pc})$.

\subsubsection{Spectral classification}

Of the final group of the 52 stars that are possible members of Cen OB1, and the 4 stars that are possible members of NGC 4755, blue spectral images of 8 stars have been obtained. We also obtained the spectrum of HD 111973, member star of NGC 4755 according to Kharchenko et al. (2004). The SpT of those stars which do not have their spectra were obtained from Kharchenko \& Roeser (2009) and Pickles \& Depagne (2010), and the radial velocity of these same stars was obtained from Kharchenko et al. (2007). The spectral classification of these nine stars, was made using the spectral classification criteria of Walborn \& Fitzpatrick (1990, hereafter WF90) Catalogue. Figures 6-9, show the spectra of these stars without HD 112364 (multiple system). Using an IRAF task it was possible to present the spectra of the REOSC Cassegrain echelle in this way. The features indicated in the spectra are the classification criteria used in these SpT.

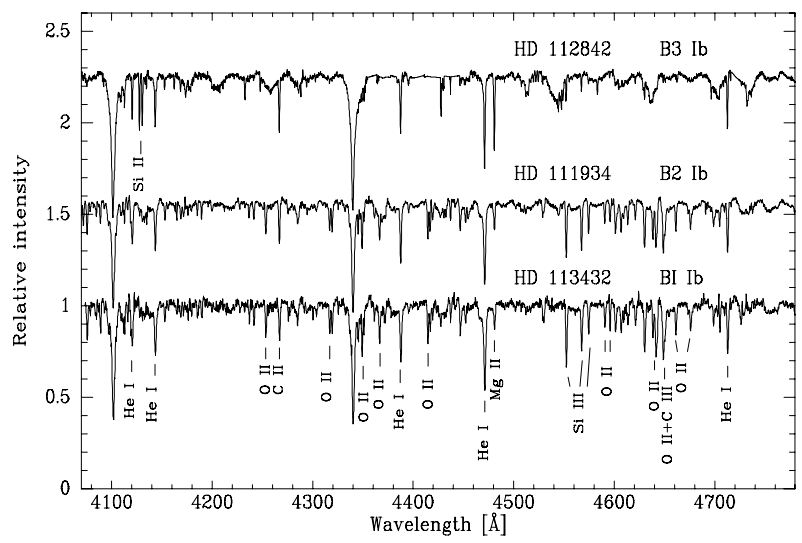

Fig. 7. Blue optical spectra of two B supergiant stars members of Cen OB1 and the B supergiant star, HD 111934, member of NGC 4755. The features indicated are the classification criteria used in the SpTs by the WF90 Catalogue.

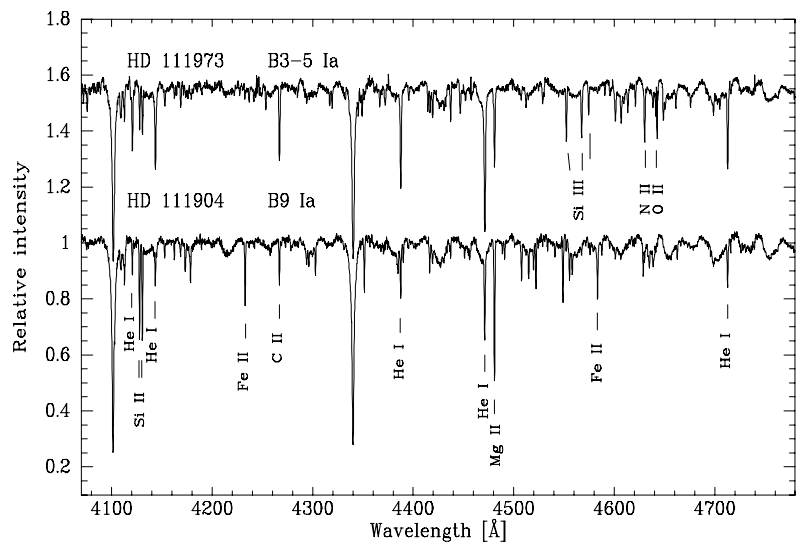

Fig. 8. Blue optical spectra of HD 111973 and HD 111904, both B supergiant stars, members of Cen OB1 and NGC 4755, respectively. The features indicated are the classification criteria used in the SpTs by the WF90 Catalogue.

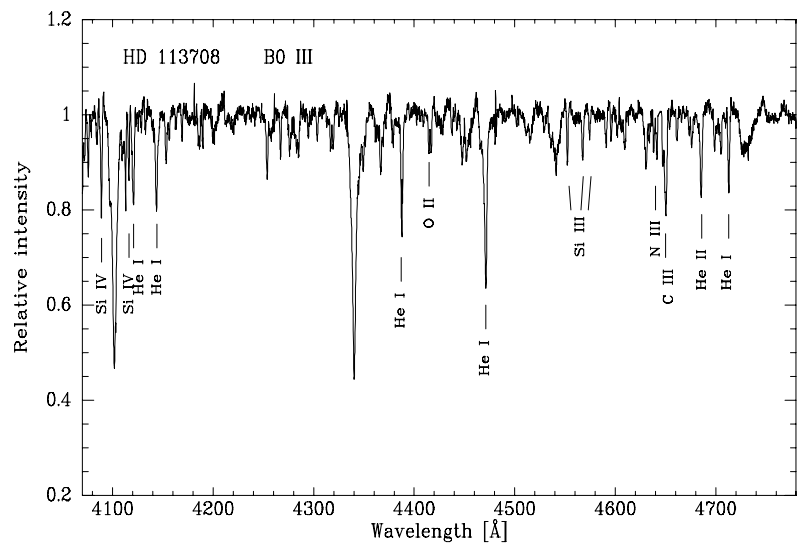

Fig. 9. Blue optical spectra of HD 113708, a B giant star, member of Cen OB1. The features indicated are the classification criteria used in the SpT by the WF90 Catalogue.

As the spectral resolution of our data $\left(0.16 \AA \mathrm{px}^{-1}\right)$ was higher than the WF90 Catalogue $\left(0.5 \AA \mathrm{px}^{-1}\right)$, the spectra of several stars of this catalogue were also obtained using the same instrumental configuration used in the observation of the stars under study. We obtained the 24 spectra of the WF90 Catalogue with better resolution. Stars observed at CASLEO were reclassified (see Col. 6 in Table 5), changing the SpT of 2 of them 
M. A. Corti and R. B. Orellana: Members of Centaurus OB1 and NGC 4755: new spectroscopic and astrometric studies
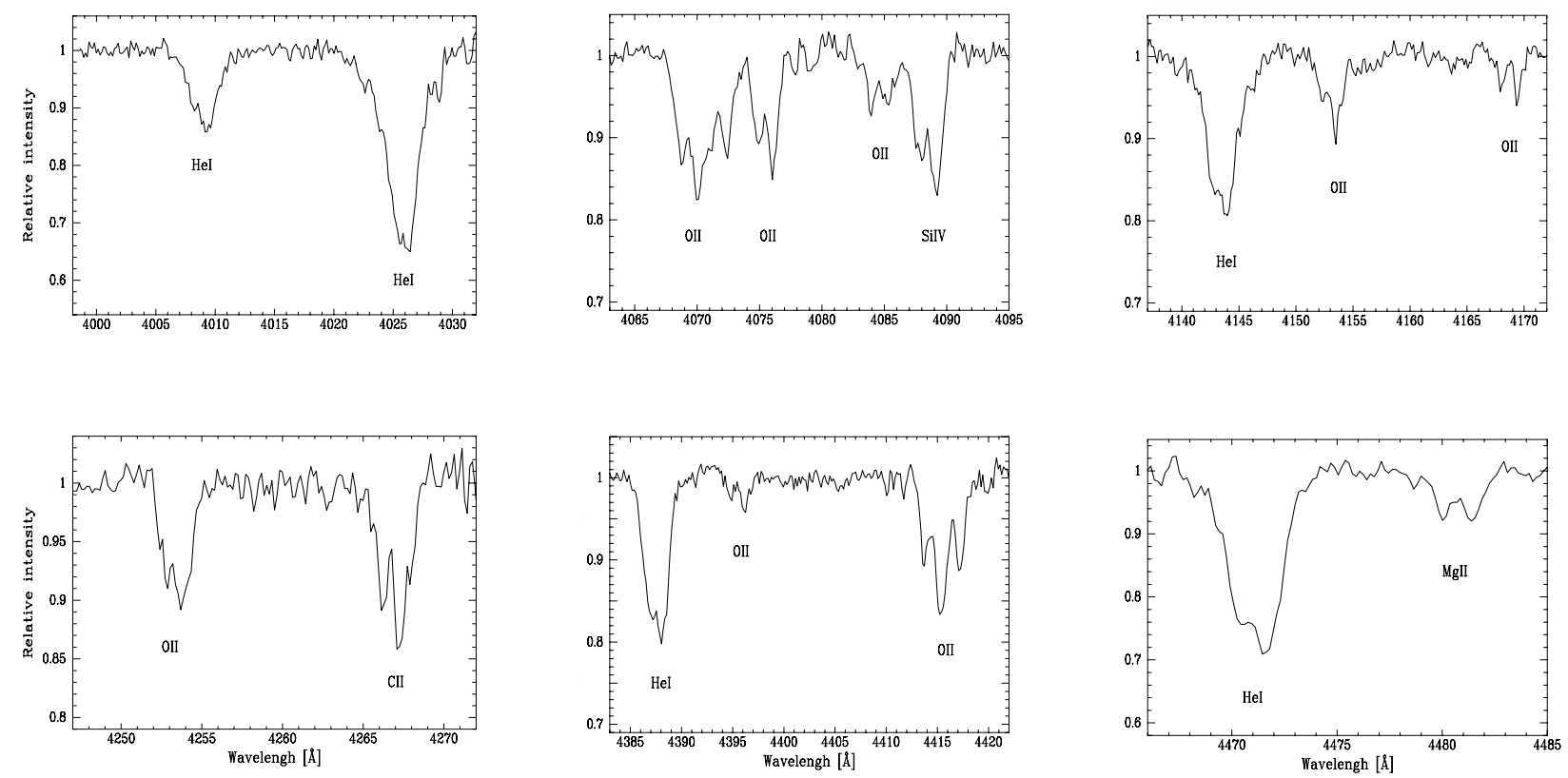

Fig. 10. Absorption lines with double and triple profiles of HD 112364. This is a binary system of two spectra (SB2).

(see Sect. 4.2). From the set of 52 stars, there are 49 stars classified as early $(\mathrm{OB})$ type and 3 stars with $\mathrm{SpT}=\mathrm{A} 0 \mathrm{Ib}-\mathrm{II}, \mathrm{SpT}=$ F0 II, and SpT = M3 II each. Among the OB type, there are 7 O-type stars ( 3 of luminosity class $(\mathrm{LC})=\mathrm{V}, 3$ of LC $=$ III, and 1 of $\mathrm{LC}=\mathrm{II}), 42$ B-type stars $(2$ of $\mathrm{LC}=\mathrm{V}, 2$ of $\mathrm{LC}=\mathrm{IV}, 2$ of $\mathrm{LC}=$ III-IV or LC $=$ III-V, 19 of LC $=$ III or LC $=$ II-III, 7 of LC = II, 3 of LC $=$ I-II, and 7 of LC $=$ Ia or Ib or Ia-b).

The HD 112364 star is a multiple-system member of Cen OB1. Figure 10 shows some of the absorption lines with a double feature of this star. The double feature are evident in all lines of He I among $\lambda 4009$ and $\lambda 4921 ; \operatorname{Mg}$ III $\lambda 4038$;

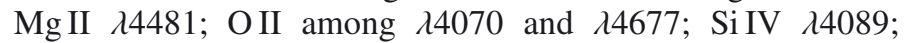
Si III $\lambda 4553, \lambda 4568, \lambda 4575, \lambda 5219$, and $\lambda 5740$. This is a binary system of two spectra (SB2). The spectral profile of OII $\lambda 4415-4417$ is triple because the opposing lines of the two spectra are added in the middle; the same addition occurs with the Mg II $\lambda 4481$ line. Only two spectra of this star could be obtained and they show the presence of the He II $\lambda 4685$ profile and the very weak absorption of the N II $\lambda 3995$ line.

The stars HD 113754 and CPD-60 4558 are presented in Kharchenko \& Roeser (2009) without LC; for this reason in this paper it was decided to assume that they are main sequence stars.

\subsubsection{Stellar radial velocities}

Using the IRAF standard reduction package, the Gaussian profile was adjusted to the lines of the different ions in the spectrum of each star. Then, to find the radial velocity of the star, only the lines of He I and He II (where they are present) were averaged for each spectrum of each star. In those stars for which it was possible to get more of one spectrum, the values of the radial velocity with their corresponding errors obtained in each spectrum were averaged. Column 10 in Table 5 lists the heliocentric radial velocity obtained for 14 possible members of the association Cen OB1. Among these 14 stars, we obtained the results of the radial velocity for 6 stars and for the other 8 stars we consulted the work of Kharchenko et al. (2007). Each radial velocity result is indicated with its corresponding error in parentheses.

The HD 112364 system mentioned in the previous section shows two sets of lines for the same ion in each of the two spectra

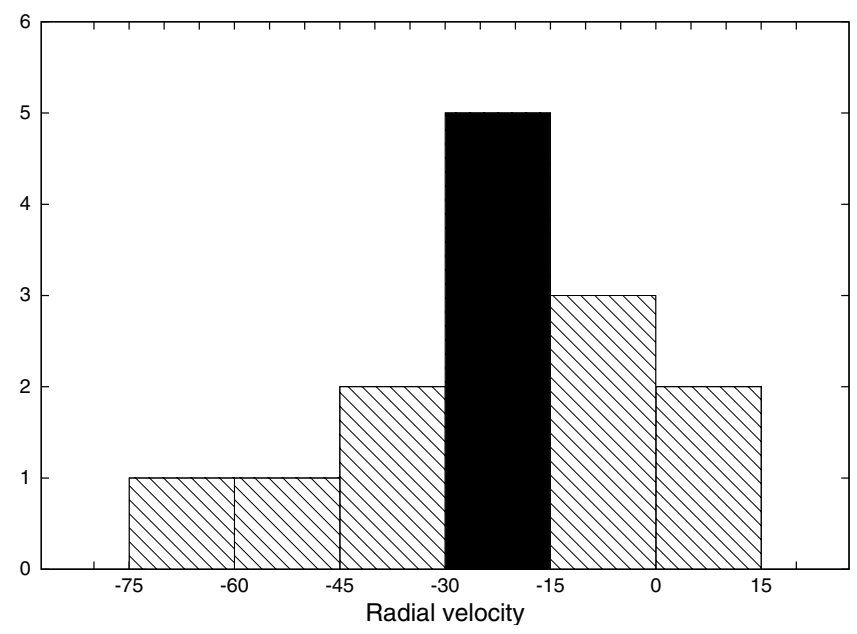

Fig. 11. Distribution of heliocentric radial velocity of the member stars of Cen OB1. The dark colour bin has the maximum value at about $-20 \mathrm{~km} \mathrm{~s}^{-1}$, the probable radial velocity of the association.

obtained for the star. The heliocentric radial velocity obtained with the He I and He II lines is $-69 \pm 8$ and $20 \pm 5 \mathrm{~km} \mathrm{~s}^{-1}$, respectively, in one spectrum, and $-61 \pm 12$ and $21 \pm 6 \mathrm{~km} \mathrm{~s}^{-1}$, respectively, in the other spectrum. With a resolution of $0.32 \AA$ every 2 pixels, and with an uncertainty of $10 \%$ in the radial velocity, the instrumental error will be about $2 \mathrm{~km} \mathrm{~s}^{-1}$, so the error with which the average values of radial velocity have been obtained is very good. The time difference between the two spectra is 3 days and the radial velocity in both spectra is similar. So, it is likely to be a system of at least two supergiants with a long period. This hypothesis is consistent with the fact that there is no evidence of interaction between the two supergiants, such as emission lines. If both components are of similar mass, we can guess a systemic velocity of about $-25 \mathrm{~km} \mathrm{~s}^{-1}$.

Figure 11 is a histogram with the distribution of all the stars that are possible members of Cen OB1, according to the heliocentric radial velocity of these stars. The width chosen for the bin was $15 \mathrm{~km} \mathrm{~s}^{-1}$ because of the average error for each star in 


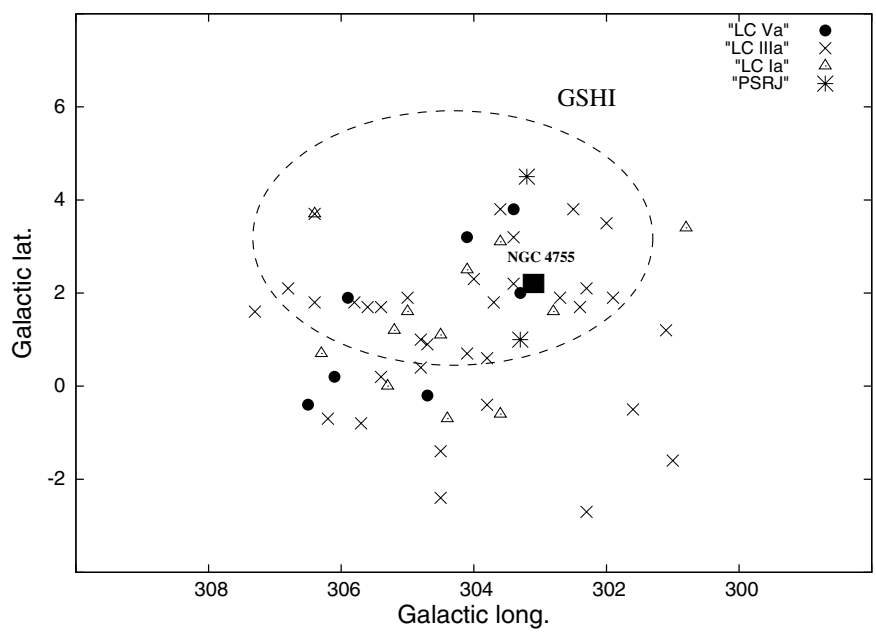

Fig. 12. Members of Cen $\mathrm{OB} 1$ in galactic coordinates. The luminous classes of the stars are indicated with different symbols (upper-right corner). The asterisks mark the location of the pulsars. The dashed ellipse represents the HI shell GS305+04-26 (Corti et al. 2012). The NGC 4755 cluster is indicated with a black box.

radial velocity so that each star would be in the appropriate bin. The largest number of stars is in the bin with a heliocentric radial velocity of $-20 \mathrm{~km} \mathrm{~s}^{-1}$.

The radial velocity of each member of NGC 4755 is shown in Table 3 and the radial velocity of the cluster was obtained averaging the radial velocity value of each of its members, which turned out to be $-18 \pm 2 \mathrm{~km} \mathrm{~s}^{-1}$.

\subsubsection{Size and age}

As can be seen in Fig. 12, the members found for the association are distributed in an almost circular area of $6^{\circ}$ in diameter. At the distance of $2600 \mathrm{pc}$ adopted for the association, it would have a linear size of $270 \mathrm{pc}$ in diameter. This size is consistent with the typical sizes traditionally accepted for the stellar association (Efremov et al. 1987).

In Fig. 12 it is possible to see that several members of Cen OB1 are in the galactic plane of our Galaxy. According to the research about the HI shell GS305+04-26, published in Corti et al. (2012) the possible generators of this HI shell were the members of Cen OB1, through their winds. Some members of Cen OB1 and the NGC 4755 cluster are inside the HI, and it has two supergiant members (one of them a possible binary system) and one giant member sharing the location with the members of the association. The location of the stars inside the shell may be a result of the action of the depletion of hydrogen because of the star formation and of the fact that the winds of all stars that are in it have swept away much of that hydrogen since several of these stars could have been formed before the shell.

To know the age of the member stars of the association, we place them in a colour-magnitude diagram (CMD) with isochrones. Figure 13 shows the comparison of the intrinsic visual magnitude and the intrinsic colour index of the stars according to its SpT (Schmidt-Kaler 1982) with theoretical isochrones for solar metallicity, mass loss, and overshooting (Marigo et al. 2008). Some scatter observed with the main sequence stars may possibly be caused by some of them belonging to multiple systems, being fast rotators, or being stars with emission. Therefore, since it was necessary to propose more than one isochrone as a solution, we employed the isochrones of 1 and $3 \times 10^{6}$ years old. A similar effect is observed with the giant and supergiant

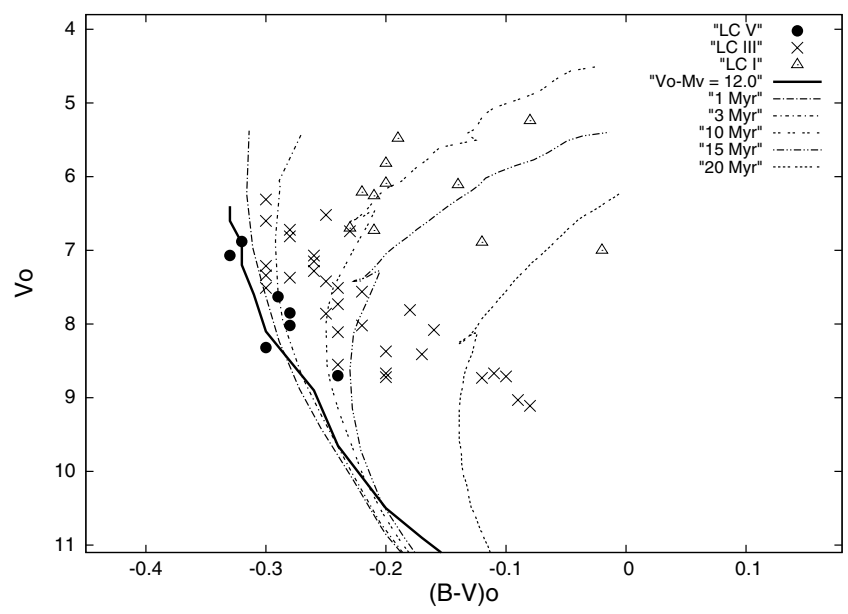

Fig. 13. CMD of member stars of Cen OB1. The symbols are the same as in Fig. 12. The black curve is the Schmidt-Kaler (1982) HR diagram for early stars; the other curves are Marigo et al. (2008) isochones for $z=0.02$. All the reference curves are corrected by an apparent distance modulus of 12.0.

stars; for these stars the isochrones of 10,15 , and $20 \times 10^{6}$ years old were employed. The fact to be possible to adjust more than one isochrones to the members of the Association could be a consequence of the star belonging to a multiple system or of a lack of precision in the determination of the luminosity class; the star that is farther away from the $20 \times 10^{6}$ year-old isochrone has a LC $=$ Ib-II. The age that we assigned to Cen OB 1 is the isochrone corresponding to the best fit to the older stars; it is $20 \times 10^{6}$ years old.

Despite the small number of members found for the cluster and the mass range between them, it is possible to know their age by the use of isochrones. To the four stars that we found to be members of NGC 4755 we added the stars TYC 8989-3110-1 and TYC 8989-1997-1, found to be members of the cluster by Kharchenko et al. (2004). With this total of six members, the age adopted for NGC 4755 is $15 \times 10^{6}$ years old.

\section{Discussion}

\subsection{Membership}

The mean proper motion of NGC 4755 that we found is in good agreement with that reported in other works (Table 1). We compared our members, shown in Table 3, with the result obtained from Humphreys (1978), Baumgardt et al. (2000), and Kharchenko et al. (2004). We found four common member stars with Kharchenko et al. (2004). A similar analysis shows that there are only two member stars of the cluster in common with Baumgardt et al. (2000) and two other stars in common with Humphreys (1978).

Only those astrometric member stars whose distance lay within $\pm 1 \sigma$ of the mean distance were considered to be member of Cen OB1. The mean spectrophotometric distance of this group turns out to be $2591 \pm 458 \mathrm{pc}(\mathrm{DM}=12.0 \pm 0.4)$. This result is in good agreement with previous Cen OB1 distance estimates (Humphreys 1978; Humphreys \& McElroy 1984). Our listing of 52 stars (see Table 5) has 19 stars in common with the Humphreys \& McElroy (1984) list of 106 stars. Within this subgroup of 19 stars, there are 10 stars that are in the Humphreys (1978) paper. 
Bearing in mind that the Tycho-2 Catalogue is $90 \%$ complete down to a visual $(V)$ magnitude $V=11.5$, we should be able to identify all supergiant stars, regardless of their SpT, that may belong to Cen OB1, and all main sequence and giant stars whose SpTs are earlier than B3 V and B6 III, respectively.

In these new spectroscopic and astrometric studies we found 56 members of Cen OB1 and NGC 4755, while in Corti et al. (2012) 54 members of Cen OB1 and NGC 4755 were found, so between both works there are 43 members in common. For this reason the total energy supplied to the interstellar medium by this new group of members of Cen OB 1 and NGC 4755 is $17 \%$ lower than the energy published in Corti et al. (2012). With the contribution of energy to the HI shell GS305+04-26 given by the pulsars, it is sufficient to generate this HI shell, as was stated in Corti et al. (2012).

The stars TYC 8990-2411-1 and TYC 8994-4147-1 with F0 II and M3 II, respectively, were found to be likely members of the association in this present paper. Their proper motions with 0.81 and 0.85 probability of belonging to Cen OB1 and $3.1 \pm 0.8$ and $2.4 \pm 0.8 \mathrm{kpc}$ of distances, respectively, make them probable members of Cen OB1. The problem with these stars is that with their parameters $(B-V)_{\mathrm{o}}=0.3$ and $V_{\mathrm{o}}=10$, and $(B-V)_{\mathrm{o}}=1.6$ and $V_{\mathrm{o}}=9.3$, respectively, locating them in Fig. 13 is impossible. They are located far from the members of the association in the CMD, so it is impossible to determine one isochrone age for them. The difficulty that arises when one wants to locate these stars in an isochrone age, reduces the likelihood of these stars being members of Cen OB1.

We find that the star WR 48 is not a member of Cen OB1 since its proper motion $\mu_{\alpha} \cos \delta=-1.9 \pm 1.3$ mas yr$^{-1}$ and $\mu_{\delta}=$ $19.0 \pm 1.8$ mas yr$^{-1}$ gives a probability of belonging $P_{i}=0$. This result disagrees with the one published by McClure-Griffiths et al. (2001).

\subsection{Spectral classification}

Among the nine stars observed at CASLEO, there is HD 111973 , known as Kappa Crucis. This star was published by Evans et al. (2005) with B3 Iae because of the emission present in the $\mathrm{H}_{\alpha}$ line $(\lambda=6562.81 \AA)$. The wavelength range covered by our spectra goes up to $6500 \AA$; so in the two spectra obtained for this star, it was not possible to observe the emission in the $\mathrm{H}_{\alpha}$ line; no emission in any other profile of these spectra was observed. For these reasons, the SpT given for this star in this work is B3-5 Ia (without emission) as Kharchenko \& Roeser (2009) and Glebocki \& Gnacinski (2005) proposed.

The spectral characteristics observed in HD 112364 (Sect. 3.2.2) are typical of a star with B0.5 Ib-a instead of B1 III (Wright et al. 2003) or B1 Ib (Garrison et al. 1977). There has been a web search on the information presented about the star HD 112364 but it is not presented anywhere as a multiple system. Even the HIPPARcos Catalogue with multiple systems (Perryman \& ESA 1997) does not have it listed. In the spectra of this star it can be seen that both components are similar, so this is probably a multiple system of supergiant stars. It will be necessary to obtain more HD 112364 spectra to know the different parameters of this system, among these the number of members of this multiple system.

The star HD 111934 was reported as a binary system in the Tycho-2 Catalogue. From the study of the two spectra obtained for this star in CASLEO, it is not possible to confirm that it is a binary system. Perhaps it is a long-period binary system, because of which the radial velocity measured in the spectra obtained
Table 4. IRAS sources possibly associated with Centaurus OB1.

\begin{tabular}{ccrccc}
\hline \hline IRAS & $\begin{array}{c}l \\
\left({ }^{\circ}\right)\end{array}$ & $\begin{array}{r}b \\
\left({ }^{\circ}\right)\end{array}$ & $\begin{array}{c}R V_{\text {LSR }} \\
\left(\mathrm{km} \mathrm{s}^{-1}\right)\end{array}$ & $\begin{array}{c}d \\
(\mathrm{kpc})\end{array}$ & Associated star \\
\hline $13024-6158$ & 304.6 & 0.6 & -27 & 2.8 & $(\ldots)$ \\
$13120-6310$ & 305.6 & -0.7 & -25 & 2.6 & $(\ldots)$ \\
$12405-6238^{a}$ & 302.0 & -0.1 & -35 & 3.3 & O9.5 \\
$13080-6229^{a}$ & 305.2 & 0.0 & -33 & 2.8 & O6.5 \\
$13092-6218^{a}$ & 305.2 & 0.2 & -33 & 3.3 & O6 \\
$13111-6228^{a}$ & 305.5 & 0.0 & -37 & 3.0 & O7.5 \\
$13134-6242^{a}$ & 305.8 & -0.2 & -39 & 3.3 & O9 \\
$13180-6245^{a}$ & 306.3 & -0.3 & -24 & 1.9 & B1 \\
\hline
\end{tabular}

References. ${ }^{(a)}$ Walsh et al. (1997).

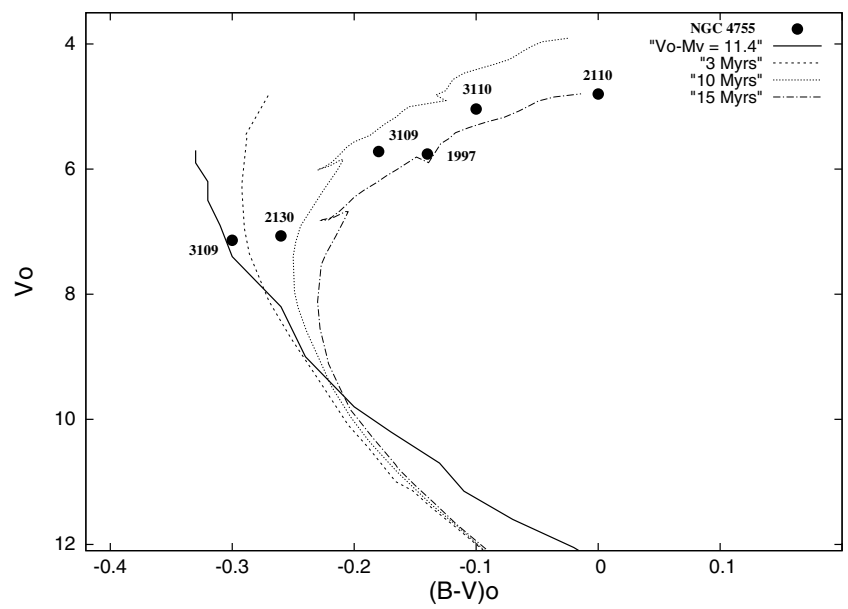

Fig. 14. CMD of member stars of NGC 4755. The symbols are the same as in Fig. 12. The black curve is the Schmidt-Kaler (1982) HR diagram for early stars; the other curves are Marigo et al. (2008) isochones for $z=0.02$. All the reference curves are corrected by an apparent distance modulus of 11.4.

with three days of difference is the same. It is also possible that both components were in the same line of sight to the observer (not quadrature) so there was a single set of lines in each spectrum. For these reasons we can't say that HD 111934 is a binary system.

We can't comment about the stars HD 112147, HD 111825 , and $\mathrm{CP}-604551$ whose spectral types are indicated with the peculiarities pe, (N) and (NE), respectively. The SpTs of these stars were obtained from the work of Kharchenko \& Roeser (2009). The spectral types of all members of NGC 4755 and Cen OB1 are presented in Tables 3 and 5, respectively.

\subsection{Stellar radial velocity}

Runaway stars are part of the examination of the kinematics of the stellar association; $\mathrm{O}$ - and B-type runaway stars are a subset of the $\mathrm{O}$ and B stars with $V_{\min } \leq\left|V_{\mathrm{pr}}\right|$, taken as $V_{\min }=30 \mathrm{~km} \mathrm{~s}^{-1}$ (Cruz-González et al. 1974). The radial component of the peculiar velocity of the star $V_{\mathrm{pr}}$ is defined as the difference between the radial velocity of the star and the radial component of the circular velocity of the star's position (both with respect to the local standard of rest (LSR); Cruz-González et al. 1974). Knowing this last parameter is necessary to be able to determine if any of these stars is a runaway star. Possible mechanisms for the origin of the OB runaways are dynamical interactions amongst members of a protocluster and as a consequence, after the supernova 


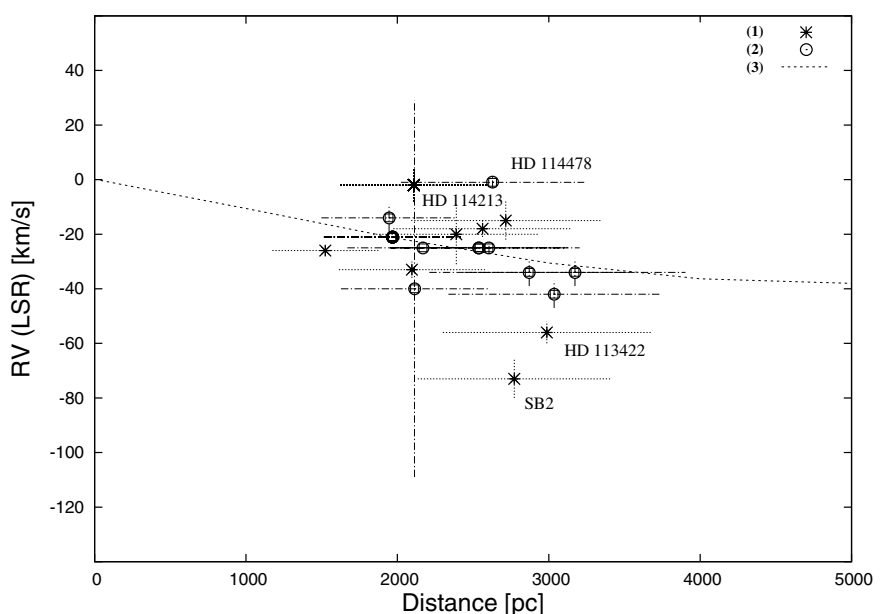

Fig. 15. AL fit of the galactic rotation model applied to our Galaxy (Fich et al. 1989). The heliocentric radial velocity is shown in Tables 3 and 5, corrected to LSR radial velocity. (1) stars of this paper; (2) stars of Kharchenko et al. (2007); (3) AL fit.

explosion of the star near this star. In order to know whether any Cen OB1 member is a runaway star, we placed the stars in the schematic diagram shown in Fig. 15. It shows the linear fit (AL) of the galactic rotation model of the galaxy (Fich et al. 1989) (the AL and the power-law fit give the same result) with the distance from the Sun and the LSR radial velocity $\mathrm{RV}_{\mathrm{LSR}}$, each parameter with its respective error bar of the possible members of Cen OB1 and NGC 4755. To locate the stars in this figure, the heliocentric radial velocity of each star was converted into the LSR radial velocity, assuming for this reason that the motion of the Sun with respect to the LSR is $13.3 \mathrm{~km} \mathrm{~s}^{-1}\left((U, V, W)_{\text {Sun }}=(9.96\right.$, $5.25,7.07) \mathrm{km} \mathrm{s}^{-1}$ ) (Aumer \& Binney 2009) towards $\alpha=18^{\mathrm{h}}$, $\delta=+30^{\circ}$.

The star farthest from the rotation model of the Galaxy is SB2 HD 112364. It is not yet possible to say anything about this star, because with the two spectra that we have it is impossible to know the barycentric radial velocity of the system. The other stars that are not coincident with the rotation model of the Galaxy are the B1 supergiants HD 113422, HD 114213, and HD 114478. In order to explain this situation we shall follow the procedure outlined by van der Sluys \& Lamers (2003) (after correcting the sign of the $\mathrm{w}_{\odot}$ term in their Eq. (19)) and Cichowolski et al. (2008) (all of this is explained in Corti et al. 2012). Using the distance from the Sun and the $\mathrm{RV}_{\mathrm{LSR}}$ of each star previously mentioned, it was possible to know the $V_{\mathrm{pr}}$ of each star. HD 113422, $V_{\mathrm{pr}}=-30 \pm 8 \mathrm{~km} \mathrm{~s}^{-1}$; HD 114213, $V_{\mathrm{pr}}=25 \pm 9 \mathrm{~km} \mathrm{~s}^{-1}$; and HD 114478, $V_{\mathrm{pr}}=27 \pm 8 \mathrm{~km} \mathrm{~s}^{-1}$. Considering one or two sigmas of each $V_{\mathrm{pr}}$ for each star, it is not possible to say that these stars are runaway stars. That is, it is possible that these stars, just like the other stars members of Cen OB1, are multiple systems or have spectra with profiles that are not well defined. In the particular case of supergiant stars, they may have instabilities in the outer atmosphere that can cause variations in the observed stellar velocity (Cruz-González et al. 1974).

In the fourth quadrant of our alaxy there is an ambiguity with the kinematic distance that corresponds to the LSR radial velocity of the object under study. We take as kinematic distances of Cen OB1 and NGC 4755 those obtained with the use of Eq. (14). The distance of Cen OB1 coincides with the kinematic distance of $2.5 \pm 0.9 \mathrm{kpc}$ from the HI shell GS305+04-26 which is associated with (Corti et al. 2012). Inserting this distance value for
Cen OB1 and the average star distance module of DM = 11.4 $(1.9 \mathrm{kpc})$ for NGC 4755 to the galactic rotation model of the Galaxy (Fich et al. 1989), it is possible to obtain an LSR radial velocity of $-26 \mathrm{~km} \mathrm{~s}^{-1}$ and $-21 \mathrm{~km} \mathrm{~s}^{-1}$, respectively. These velocities, correspond to about $-22 \mathrm{~km} \mathrm{~s}^{-1}$ and $-17 \mathrm{~km} \mathrm{~s}^{-1}$ velocity in the heliocentric system, respectively. The latter is in good agreement with the heliocentric radial velocity value found for Cen OB1 according to Sect. 3.2.3 and the value found for NGC 4755 making the average of the information presented in Table 3.

\subsection{Age}

There are two pulsars located near the HI shell GS305+04-26, PSRJ 1253-5820 is located inside the shell, and PSRJ 1254-6150 is located on the edge of the shell. Both pulsars could have been generated as a result of the explosion as $\mathrm{SNe}$ of two stars possible members of Cen OB1, which should be in the group of older members of the association (Corti et al. 2012). Several factors support this hypothesis, the age of $5 \times$ $10^{6}$ years old and the distance of $2.24 \mathrm{kpc}$ for PSRJ 1254-6150 (Manchester et al. 2005), and the age of $2 \times 10^{6}$ years old (Manchester et al. 2005) and the distance of $2.2 \mathrm{kpc}$ (Noutsos et al. 2008) for PSRJ 1253-5820.

We have also investigated the possible existence of IRAS sources in the area of the galaxy where Cen OB1 is present. For this, we consulted the paper of Walsh et al. (1997), which lists 215 candidates as UC HII regions, obtained as a result of Methanol maser of IRAS-selected sources. It consists of IRAS sources associated with early stars. They associated the LSR radial velocity of these stars with IRAS sources. Table 4 shows the LSR radial velocities of these stars (Col. 4) and the minimal distance of these stars from the Sun, obtained with the galactic rotation model of the Galaxy (Fich et al. 1989) (Col. 5). The weight of the individual distance determinations stems from the assumption of an uncertainty of $\pm 10 \mathrm{~km} \mathrm{~s}^{-1}$ caused by noncircular motion (Burton 1988), from which we can infer that the value of the distance obtained in this way has an error of $1 \mathrm{kpc}$, approximately. This makes it possible for all IRAS sources studied by Walsh et al. (1997) to share their location in the galaxy with the association.

Referring to the IRAS infrared survey published in the Narrow-band $\mathrm{H} \alpha$ data retrieved from the SuperCOSMOS H-alpha Survey (SHS) ${ }^{4}$ (Parker et al. 2005), we have found the IRAS sources 13120-6310 and 13024-6158, whose radial velocity data were obtained from Mottram et al. (2007) and Bronfman et al. (1996), respectively. Correcting their heliocentric radial velocities to the LSR system, a radial velocity of $V_{\mathrm{LSR}}=-25 \mathrm{~km} \mathrm{~s}^{-1}$ and $V_{\mathrm{LSR}}=-27 \mathrm{~km} \mathrm{~s}^{-1}$, respectively, was obtained for them. By employing the galactic rotation model of the Galaxy (Fich et al. 1989), we estimated a minimal distance to the Sun of $2.6 \mathrm{kpc}$ and $2.8 \mathrm{kpc}$, respectively.

In Sect. 3.2.4 we studied the age of the members of Cen OB1 and the result was a distribution of ages. The older stars of the association would be those that originated the pulsars that we see today. Likewise, the age of Cen OB1 is determined by the older stars to which one isochrone can be fitted, and is also about $2 \times 10^{7}$ years old. In this subsection, we have seen that near Cen OB1, IRAS sources are found, which show the youngest star formation. For all the above reasons we can think of the possibility that the region under study is a sequential star formation region. The latter may be because the Milky Way arm

4 http://www-wfau.roe.ac.uk/sss/halpha/index.html 
Carina-Sagittarius, where this association is located, is an area rich in hydrogen.

\section{Conclusions}

This work enables us to appreciate how powerful it is to investigate members of a stellar association, by employing astrometric and astrophysical techniques simultaneously. This paper shows the first and unique astrometric research about the membership of the Cen OB1. With our own data and catalogue data, new spectroscopic and astrometric studies of members of the association Cen OB1 and the open cluster NGC 4755 have been made. The result of this investigation is that Cen OB1 has 52 members and NGC 4755 has 4 members, and that it is an open cluster integrated into the Cen OB1. The latter centred at $(l, b)=$ $\left(303.7,0^{\circ} .4\right)$ is approximately circular in shape with $270 \mathrm{pc}$ of diameter. It is at $2591 \pm 458 \mathrm{pc}$ from the Sun and the heliocentric radial velocity is $\sim-20 \mathrm{~km} \mathrm{~s}^{-1}$. The components of the mean proper motion of Cen OB 1 are $\mu_{\alpha} \cos \delta=-4.78 \pm 0.10$ mas yr$^{-1}$

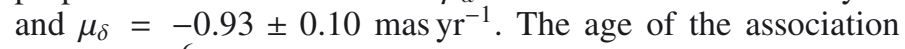
is $\sim 20 \times 10^{6}$ years old. The open cluster NGC 4755 centred at $(l, b)=\left(303^{\circ} 2,2.5\right)$ is at $1888 \pm 481 \mathrm{pc}$ from the Sun and the heliocentric radial velocity is $-18 \pm 2 \mathrm{~km} \mathrm{~s}^{-1}$. The components of its mean proper motion are $\mu_{\alpha} \cos \delta=-4.14 \pm 0.19$ mas yr$^{-1}$

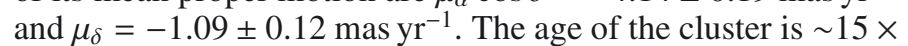
$10^{6}$ years old. There is good agreement between the distances and characteristic ages of the pulsars PSR J1253-5820 and PSR J1254-6150 with the idea that they were born as the result of SNe undergone in the past by massive members of Cen OB1. We have seen IRAS sources near Cen OB1 showing that even star formation is occurring. The age gradient observed between the different members of Cen OB1 makes it likely that the association and IRAS sources represent different stages of sequential formation process.

Acknowledgements. This work was partially supported by Consejo Nacional de Investigaciones Científicas y Técnicas CONICET under projects PIP 01299 and 01359, and by Universidad Nacional de La Plata (UNLP) under projects 11/G091 and 11/G096. We would like to thank Dr. Gustavo, Baume and Dr E. Marcelo, Arnal for helping us to prepare and to interpret the CMD (Figs. 13 and 14) and Fig. 15, respectively. One of us would like to thank Dr. Roberto, Gamen for helping with one IRAF task. We thank the referee for many helpful comments that significantly improved the manuscript.

\section{References}

Aumer, M., \& Binney, J. J. 2009, MNRAS, 397, 1286

Baumgardt, H., Dettbarn, C., \& Wielen, R. 2000, A\&AS, 146, 251

Bessell, M. S., \& Brett, J. M. 1988, PASP, 100, 1134

Bronfman, L., Nyman, L.-A., \& May, J. 1996, A\&AS, 115, 81
Brown, A. G. A., Blaauw, A., Hoogerwerf, R., de Bruijne, J. H. J., \& de Zeeuw, P. T. 1999, in The Origin of Stars and Planetary Systems, eds. C. J. Lada, \& N. D. Kylafis, NATO ASIC Proc., 540, 411

Burton, W. B. 1988, in Galactic and Extragalactic Radio Astronomy, 2nd edn. (Berlin: Springer), 295

Cichowolski, S., Pineault, S., Arnal, E. M., \& Cappa, C. E. 2008, A\&A, 478, 443

Corti, M. A., Arnal, E. M., \& Orellana, R. B. 2012, A\&A, 546, A62

Cruz-González, C., Recillas-Cruz, E., Costero, R., Peimbert, M., \& TorresPeimbert, S. 1974, Rev. Mex. Astron. Astrofis., 1, 211

Dias, W. S., Alessi, B. S., Moitinho, A., \& Lépine, J. R. D. 2002, A\&A, 389, 871

Efremov, I. N., Ivanov, G. R., \& Nikolov, N. S. 1987, Ap\&SS, 135, 119

Evans, C. J., Smartt, S. J., Lee, J.-K., et al. 2005, A\&A, 437, 467

Fich, M., Blitz, L., \& Stark, A. A. 1989, ApJ, 342, 272

Garrison, R. F., Hiltner, W. A., \& Schild, R. E. 1977, ApJS, 35, 111

Glebocki, R., \& Gnacinski, P. 2005, in The Future of Cool-Star Astrophysics: 12th Cambridge Workshop on Cool Stars, Stellar Systems, and the Sun, eds. A. Brown, G. M. Harper, \& T. R. Ayres (University of Colorado), 823, VizieR Online Data Catalog, III/244

Høg, E., Fabricius, C., Makarov, V. V., et al. 2000a, A\&A, 357, 367

Høg, E., Fabricius, C., Makarov, V. V., et al. 2000b, A\&A, 355, L27

Humphreys, R. M. 1978, ApJS, 38, 309

Humphreys, R. M., \& McElroy, D. B. 1984, catalogue of stars in stellar associations and young clusters (University of Minnesota), Vizier Online Catalog

Jones, B. F., \& Walker, M. F. 1988, AJ, 95, 1755

Kharchenko, N. V., \& Roeser, S. 2009, VizieR Online Data Catalog, I/280

Kovalevsky, J., Lindegren, L., Perryman, M. A. C., et al. 1997, A\&A, 323, 620

Kharchenko, N. V., Piskunov, A. E., Röser, S., Schilbach, E., \& Scholz, R.-D. 2004, Astron. Nachr., 325, 740

Kharchenko, N. V., Piskunov, A. E., Röser, S., Schilbach, E., \& Scholz, R.-D. 2005, A\&A, 438, 1163

Kharchenko, N. V., Scholz, R.-D., Piskunov, A. E., Röser, S., \& Schilbach, E. 2007, Astron. Nachr., 328, 889

Manchester, R. N., Hobbs, G. B., Teoh, A., \& Hobbs, M. 2005, AJ, 129, 1993

Marigo, P., Girardi, L., Bressan, A., et al. 2008, A\&A, 482, 883

McClure-Griffiths, N. M., Dickey, J. M., Gaensler, B. M., \& Green, A. J. 2001 , ApJ, 562, 424

Mottram, J. C., Hoare, M. G., Lumsden, S. L., et al. 2007, A\&A, 476, 1019

Noutsos, A., Johnston, S., Kramer, M., \& Karastergiou, A. 2008, MNRAS, 386, 1881

Orellana, R. B., de Biasi, M. S., Bustos Fierro, I. H., \& Calderón, J. H. 2010, A\&A, 521, A39

Parker, Q. A., Phillipps, S., Pierce, M. J., et al. 2005, MNRAS, 362, 689

Perryman, M. A. C., \& ESA 1997, The HIPPARCos and TYCHO catalogues. Astrometric and photometric star catalogues derived from the ESA HIPPARCOS Space Astrometry Mission, ESA SP, 1200

Pickles, A., \& Depagne, É. 2010, PASP, 122, 1437

Sanders, W. L. 1971, A\&A, 14, 226

Schmidt-Kaler, Th. 1982, In Landolt-Bornstein New Series, Group VI, Vol. 2b, eds. K. Schaifers, \& H. H. Voigt (Berlin: Springer-Verlag)

Urban, S. E., Wycoff, G. L., \& Makarov, V. V. 2000, AJ, 120, 501

van den Bergh, S., \& Sher, D. 1960, Publications of the David Dunlap Observatory, 2, 203

van der Sluys, M. V., \& Lamers, H. J. G. L. M. 2003, A\&A, 398, 181

Vasilevskis, S., Klemola, A., \& Preston, G. 1958, AJ, 63, 387

Walborn, N. R. 1972, AJ, 77, 312

Walborn, N., \& Fitzpatrick, E. 1990, PASP, 102, 379

Walsh, A. J., Hyland, A. R., Robinson, G., \& Burton, M. G. 1997, MNRAS, 291, 261

Wright, C. O., Egan, M. P., Kraemer, K. E., \& Price, S. D. 2003, AJ, 125, 359

Page 12 is available in the electronic edition of the journal at http: //www . aanda.org 
Table 5. Possible members of Centaurus OB1.

\begin{tabular}{|c|c|c|c|c|c|c|c|c|c|c|}
\hline ID & $\begin{array}{l}\alpha_{J 2000.0} \\
(\mathrm{~h}: \mathrm{m}: \mathrm{s})\end{array}$ & $\begin{array}{c}\delta_{J 2000.0} \\
\left({ }^{\circ}:^{\prime}:^{\prime \prime}\right)\end{array}$ & $\begin{array}{c}V \\
(\mathrm{mag})\end{array}$ & $\begin{array}{c}(B-V) \\
(\mathrm{mag})\end{array}$ & SpT & $\begin{array}{c}\mu_{\alpha} \cos \delta \\
\left(\mathrm{mas} \mathrm{yr}^{-1}\right)\end{array}$ & $\begin{array}{r}\mu_{\delta} \\
\left(\mathrm{mas} \mathrm{yr}^{-1}\right)\end{array}$ & TYC & $\begin{array}{c}\mathrm{RV} \\
\left(\mathrm{km} \mathrm{s}^{-1}\right)\end{array}$ & DM \\
\hline HD 112842 & $13: 00: 31.5$ & $-60: 22: 31$ & 7.1 & 0.2 & B3 $\mathrm{Ib}^{1}$ & -4.2 & -1.1 & $8989-0728-1$ & $-27(3)^{1}$ & 11.6 \\
\hline HD 112364 & $12: 56: 59.6$ & $-59: 44: 33$ & 7.4 & 0.2 & B0.7Ib ${ }^{1}$ & -5.6 & -0.6 & $8660-1247-1$ & $-67(7)^{1}$ & 12.2 \\
\hline HD 112366 & $12: 57: 11.1$ & $-63: 27: 52$ & 7.6 & 0.6 & B6 Iab- $b^{3}$ & -4.0 & -1.2 & $8993-0763-1$ & $-7(5)^{2}$ & 11.4 \\
\hline HD 109505 & $12: 35: 46.3$ & $-61: 34: 22$ & 8.0 & 0.2 & $\mathrm{~B} 2 \mathrm{II}^{3}$ & -5.1 & -1.2 & $8988-0451-1$ & $(\ldots)$ & 11.5 \\
\hline HD 113708 & $13: 06: 48.3$ & $-65: 12: 44$ & 8.1 & 0.0 & B0 III ${ }^{1}$ & -3.0 & -1.3 & $8997-2020-1$ & $-9(7)^{1}$ & 12.4 \\
\hline HD 115704 & $13: 20: 12.4$ & $-62: 00: 22$ & 8.1 & 0.4 & B0.5 $\mathrm{Iab}^{3}$ & -5.1 & 0.2 & 8994-0491-1 & $(\ldots)$ & 12.6 \\
\hline HD 114394 & $13: 11: 20.5$ & $-59: 34: 36$ & 8.2 & 0.1 & $\mathrm{~B} 1 \mathrm{III}^{3}$ & -4.3 & -0.2 & $8661-1882-1$ & (..) & 11.6 \\
\hline HD 112784 & 13:00:05.6 & $-60: 35: 37$ & 8.3 & 0.0 & $09.5 \mathrm{III}^{3}$ & -4.9 & -2.7 & $8989-1025-1$ & $-28(5)^{2}$ & 12.5 \\
\hline HD 113422 & $13: 04: 36.9$ & $-61: 42: 37$ & 8.3 & 0.7 & $\mathrm{~B} 1 \mathrm{Ia}^{1}$ & -5.3 & -1.3 & $8989-0178-1$ & $-50(4)^{1}$ & 12.4 \\
\hline HD 112497 & $12: 58: 00.9$ & $-61: 03: 19$ & 8.4 & 0.3 & $\mathrm{~B} 1-2 \mathrm{II}-\mathrm{III}^{3}$ & -4.0 & 0.3 & $8989-1412-1$ & (..) & 11.6 \\
\hline HD 113606 & 13:06:03.1 & $-64: 14: 35$ & 8.7 & 0.3 & $09.5 \mathrm{III}^{3}$ & -5.3 & 0.8 & 8997-1199-1 & $(\ldots)$ & 12.0 \\
\hline HD 114478 & $13: 11: 57.4$ & $-62: 48: 54$ & 8.7 & 0.4 & $\mathrm{~B} 1 \mathrm{Ib}-\mathrm{II}^{3}$ & -6.4 & -0.7 & $8994-1288-1$ & $6(2)^{2}$ & 12.1 \\
\hline HD 112026 & $12: 54: 18.4$ & $-60: 53: 39$ & 8.7 & 0.0 & $\mathrm{~B} 0-1 \mathrm{IV}^{3}$ & -4.7 & -1.2 & $8989-2291-1$ & $(\ldots)$ & 12.5 \\
\hline HD 115223 & $13: 16: 52.5$ & $-60: 27: 23$ & 8.7 & 0.5 & $\mathrm{~A} 0 \mathrm{Ib}-\mathrm{II}^{3}$ & -5.4 & 0.8 & $8990-1797-1$ & $(\ldots)$ & 12.2 \\
\hline CP-60 4528 & $13: 13: 11.7$ & $-61: 05: 21$ & 8.8 & 0.2 & $\mathrm{~B} 1 \mathrm{III}^{3}$ & -4.1 & -0.5 & $8990-2216-1$ & $-19^{2}$ & 11.7 \\
\hline HD 112181 & $12: 55: 31.8$ & $-60: 39: 00$ & 8.8 & 0.0 & $\mathrm{~B} 2-3 \mathrm{II}-\mathrm{III}^{3}$ & -4.2 & -2.4 & 8989-0473-1 & (...) & 12.6 \\
\hline HD 113432 & $13: 04: 49.3$ & $-63: 29: 29$ & 8.9 & 0.6 & $\mathrm{~B} 1 \mathrm{Ib}^{1}$ & -3.5 & -1.7 & $8993-1371-1$ & $-14(11)^{1}$ & 11.9 \\
\hline HD 114213 & $13: 10: 21.3$ & $-61: 28: 20$ & 9.0 & 0.8 & $\mathrm{~B} 1 \mathrm{Ia}-\mathrm{b}^{1}$ & -3.9 & -2.6 & $8990-2881-1$ & $4(6)^{1}$ & 11.6 \\
\hline HD 116403 & $13: 24: 51.6$ & $-61: 01: 44$ & 9.0 & 0.0 & B5 II-III ${ }^{3}$ & -4.9 & -2.2 & $8990-2566-1$ & (...) & 12.4 \\
\hline HD 110984 & $12: 46: 44.8$ & $-61: 11: 11$ & 9.0 & 0.3 & $\mathrm{~B} 1 \mathrm{II}-\mathrm{III}^{3}$ & -4.4 & -2.5 & $8988-2898-1$ & (...) & 12.5 \\
\hline HD 112382 & $12: 56: 59.9$ & $-59: 02: 00$ & 9.0 & 0.2 & $\mathrm{~B} 4 \mathrm{II}^{3}$ & -4.6 & -1.5 & $8660-1349-1$ & $(\ldots)$ & 12.0 \\
\hline HD 114669 & $13: 13: 10.7$ & $-62: 31: 38$ & 9.1 & 0.1 & $\mathrm{~B} 5 \mathrm{II}^{3}$ & -4.7 & 0.0 & 8994-2693-1 & $(\ldots)$ & 12.1 \\
\hline HD 113014 & $13: 01: 48.1$ & $-62: 10: 52$ & 9.1 & 0.2 & B2 III $^{3}$ & -4.2 & -1.6 & 8993-0329-1 & $(\ldots)$ & 11.4 \\
\hline HD 114530 & $13: 12: 15.3$ & $-61: 04: 16$ & 9.1 & 0.2 & $\mathrm{O} 9.5 \mathrm{III}^{3}$ & -5.0 & -1.9 & $8990-1060-1$ & $(\ldots)$ & 12.6 \\
\hline HD 111579 & $12: 51: 03.5$ & $-61: 14: 37$ & 9.1 & 0.5 & $\mathrm{~B} 2 \mathrm{Ib}-\mathrm{II}^{3}$ & -5.6 & -0.6 & $8988-3009-1$ & $(\ldots)$ & 12.4 \\
\hline HD 112147 & $12: 55: 08.4$ & $-59: 01: 07$ & 9.1 & 0.2 & B0 IV pe ${ }^{3}$ & -5.9 & -0.6 & $8660-2400-1$ & (...) & 12.3 \\
\hline CPD-61 3462 & $13: 07: 33.0$ & $-62: 22: 17$ & 9.2 & 0.4 & $\mathrm{~B} 0.5 \mathrm{II}^{3}$ & -4.4 & -1.2 & $8993-0530-1$ & $-36(5)^{2}$ & 12.4 \\
\hline HD 113742 & $13: 06: 53.7$ & $-61: 56: 38$ & 9.2 & 0.3 & $\mathrm{~B} 1-2 \mathrm{III}^{3}$ & -3.7 & -0.6 & $8993-0120-1$ & (...) & 11.8 \\
\hline HD 116121 & $13: 23: 03.7$ & $-63: 03: 42$ & 9.3 & 0.0 & $09.5 \mathrm{~V}^{3}$ & -6.3 & -0.3 & 8994-2386-1 & (...) & 12.6 \\
\hline HD 112661 & $12: 59: 21.2$ & $-62: 17: 20$ & 9.3 & 0.5 & B0-1 III-IV ${ }^{3}$ & -2.6 & -1.0 & $8993-0176-1$ & (...) & 11.6 \\
\hline CP-61 3452 & $13: 06: 48.9$ & $-61: 47: 00$ & 9.3 & 0.6 & $\mathrm{O} 9.5 \mathrm{II}^{3}$ & -6.1 & -1.1 & 8989-0004-1 & (..) & 12.1 \\
\hline HD 114011 & $13: 08: 43.0$ & $-61: 11: 10$ & 9.3 & 0.6 & $\mathrm{~B} 4 \mathrm{Ib}-\mathrm{II}^{3}$ & -5.3 & -1.1 & $8990-2510-1$ & $-28(5)^{2}$ & 12.3 \\
\hline HD 110449 & $12: 42: 52.9$ & $-60: 58: 32$ & 9.3 & 0.1 & B2 III $^{3}$ & -4.7 & -0.6 & $8988-2659-1$ & (..) & 12.0 \\
\hline HD 115746 & $13: 20: 35.5$ & $-63: 24: 43$ & 9.4 & 0.2 & B2-4 III-V ${ }^{3}$ & -3.8 & -0.5 & $8994-3012-1$ & (...) & 11.6 \\
\hline HD 115666 & $13: 19: 54.4$ & $-60: 52: 20$ & 9.4 & 0.1 & $\mathrm{~B} 7 \mathrm{II}^{3}$ & -6.3 & -1.7 & $8990-0696-1$ & (...) & 12.2 \\
\hline HD 109937 & $12: 39: 19.4$ & $-63: 21: 41$ & 9.4 & 0.3 & B2-3 $\mathrm{III}^{3}$ & -4.2 & -0.7 & $8992-0460-1$ & (...) & 11.5 \\
\hline CPD-62 3113 & $13: 16: 28.4$ & $-63: 30: 31$ & 9.4 & 1.6 & M3 II $^{4}$ & -3.9 & -0.4 & $8994-4147-1$ & $(\ldots)$ & 11.9 \\
\hline HD 111825 & $12: 52: 45.9$ & $-60: 48: 36$ & 9.4 & 0.0 & $\mathrm{~B} 1-3 \mathrm{~V}(\mathrm{~N})^{3}$ & -5.3 & -1.2 & $8989-2903-1$ & (...) & 11.9 \\
\hline CPD-60 4558 & $13: 15: 53.4$ & $-60: 49: 34$ & 9.5 & 0.4 & $\mathrm{O}^{3}$ & -5.6 & -0.4 & $8990-0828-1$ & (...) & 12.6 \\
\hline HD 110597 & $12: 43: 53.0$ & $-59: 20: 09$ & 9.5 & 0.1 & $\mathrm{~B} 8 \mathrm{II}^{3}$ & -3.7 & 0.1 & $8659-2930-1$ & (..) & 11.8 \\
\hline HD 113754 & $13: 07: 04.4$ & $-62: 59: 30$ & 9.5 & 0.5 & $06-7^{3}$ & -2.9 & 0.0 & $8993-0558-1$ & $-19(2)^{2}$ & 12.1 \\
\hline HD 113991 & $13: 08: 31.1$ & $-60: 56: 00$ & 9.5 & 0.1 & B3 III $^{3}$ & -4.4 & -2.1 & 8989-0880-1 & (..) & 11.4 \\
\hline CP-61 3576 & $13: 18: 39.2$ & $-62: 31: 48$ & 9.5 & 0.2 & B $0.5 \mathrm{~V}^{3}$ & -3.9 & -0.1 & $8994-0182-1$ & $-34(69)^{2}$ & 11.6 \\
\hline HD 109253 & $12: 33: 58.7$ & $-64: 22: 36$ & 9.6 & 0.2 & B8 II-III ${ }^{3}$ & -4.7 & -2.2 & $8983-1501-1$ & (..) & 11.8 \\
\hline HD 111121 & $12: 47: 47.1$ & $-59: 05: 04$ & 9.6 & 0.1 & B8-9 II- III $^{3}$ & -5.9 & 0.9 & $8659-1977-1$ & (...) & 12.1 \\
\hline HD 112637 & $12: 59: 15.6$ & $-63: 18: 16$ & 9.6 & 0.3 & $\mathrm{~B} 2 \mathrm{III}^{3}$ & -5.3 & -2.5 & $8993-0073-1$ & $(\ldots)$ & 11.6 \\
\hline HD 111377 & $12: 49: 37.7$ & $-60: 57: 56$ & 9.6 & 0.1 & B2-5 II-III ${ }^{3}$ & -4.9 & -2.9 & $8988-1854-1$ & $(\ldots)$ & 11.7 \\
\hline HD 110864 & $12: 45: 56.5$ & $-60: 46: 21$ & 9.6 & 0.1 & B3 II-III ${ }^{3}$ & -5.2 & 0.1 & $8988-2236-1$ & $(\ldots)$ & 11.7 \\
\hline (...) & $13: 22: 49.1$ & $-60: 30: 12$ & 9.7 & 0.2 & $\mathrm{FO} \mathrm{II}^{4}$ & -6.0 & -0.6 & $8990-2411-1$ & $(\ldots)$ & 12.5 \\
\hline CD-64 654 & $12: 45: 44.1$ & $-65: 37: 23$ & 9.7 & 0.1 & $\mathrm{~B} 2 \mathrm{III}^{3}$ & -3.8 & -1.0 & $8996-0235-1$ & $(\ldots)$ & 12.4 \\
\hline HD 115497 & $13: 18: 40.7$ & $-59: 00: 39$ & 9.7 & 0.1 & B8-9 II $^{3}$ & -6.2 & 0.5 & $8661-1030-1$ & $(\ldots)$ & 12.2 \\
\hline CP-60 4551 & $13: 15: 10.0$ & $-60: 58: 01$ & 9.9 & 0.5 & $\mathrm{~B} 1 \mathrm{III}(\mathrm{NE})^{3}$ & -4.7 & 0.1 & $8990-1366-1$ & $(\ldots)$ & 11.9 \\
\hline
\end{tabular}

References. ${ }^{(1)}$ This paper. ${ }^{(2)}$ Kharchenko et al. (2007). ${ }^{(3)}$ Kharchenko \& Roeser (2009). ${ }^{(4)}$ Pickles \& Depagne (2010). 